\title{
Convenient Synthesis of Benziodazolone: New Reagents for Direct Esterification of Alcohols and Amidation of Amines
}

\author{
Michael T. Shea ${ }^{1}$, Gregory T. Rohde ${ }^{2}$ D, Yulia A. Vlasenko ${ }^{3}$, Pavel S. Postnikov ${ }^{3} \mathbb{D}$, Mekhman S. Yusubov $^{3}$, \\ Viktor V. Zhdankin $1, * \mathbb{D}$, Akio Saito ${ }^{4, *(\mathbb{D})}$ and Akira Yoshimura $1,3, * \mathbb{D}$ \\ 1 Department of Chemistry and Biochemistry, University of Minnesota Duluth, Duluth, MN 55812, USA; \\ sheax152@d.umn.edu \\ 2 Marshall School, Duluth, MN 55811, USA; greg.rohde@marshallschool.org \\ 3 Research School of Chemisty and Applied Biomediacl Sciences, The Tomsk Polytechnic University, \\ 634050 Tomsk, Russia; yav6@tpu.ru (Y.A.V.); postnikov@tpu.ru (P.S.P.); yusubov@mail.ru (M.S.Y.) \\ 4 Division of Applied Chemistry, Institute of Engineering, Tokyo University of Agriculture and Technology, \\ Tokyo 184-8588, Japan \\ * Correspondence: vzhdanki@d.umn.edu (V.V.Z.); akio-sai@cc.tuat.ac.jp (A.S.); yoshimura@tpu.ru (A.Y.)
}

\section{check for} updates

Citation: Shea, M.T.; Rohde, G.T.; Vlasenko, Y.A.; Postnikov, P.S.;

Yusubov, M.S.; Zhdankin, V.V.; Saito,

A.; Yoshimura, A. Convenient

Synthesis of Benziodazolone: New Reagents for Direct Esterification of Alcohols and Amidation of Amines. Molecules 2021, 26, 7355. https:// doi.org/10.3390/molecules26237355

Academic Editors: Irina A. Balova and Alexander S. Antonov

Received: 22 November 2021

Accepted: 2 December 2021

Published: 3 December 2021

Publisher's Note: MDPI stays neutral with regard to jurisdictional claims in published maps and institutional affiliations.

Copyright: (c) 2021 by the authors. Licensee MDPI, Basel, Switzerland. This article is an open access article distributed under the terms and conditions of the Creative Commons Attribution (CC BY) license (https:/ / creativecommons.org/licenses/by/ $4.0 /)$.

\begin{abstract}
Hypervalent iodine heterocycles represent one of the important classes of hypervalent iodine reagents with many applications in organic synthesis. This paper reports a simple and convenient synthesis of benziodazolones by the reaction of readily available iodobenzamides with $m$-chloroperoxybenzoic acid in acetonitrile at room temperature. The structure of one of these new iodine heterocycles was confirmed by $\mathrm{X}$-ray analysis. In combination with $\mathrm{PPh}_{3}$ and pyridine, these benziodazolones can smoothly react with alcohols or amines to produce the corresponding esters or amides of 3-chlorobenzoic acid, respectively. It was found that the novel benziodazolone reagent reacts more efficiently than the analogous benziodoxolone reagent in this esterification.
\end{abstract}

Keywords: hypervalent iodine; iodine heterocycles; benziodazolone; aroylation

\section{Introduction}

Iodine(III)-containing heterocycles represent one of the most important classes of hypervalent iodine reagents [1-8]. Among them, the five-membered iodine-oxygen heterocycles, which are known by the general name of benziodoxolones, have found wide application in organic synthesis [9-14]. In particular, benziodoxolone derivatives 1 (Figure 1) have attracted significant interest as atom-transfer reagents and oxidants [5,10-14]. Recently, benziodoxolone derivatives have been used as effective oxidants for coupling of carboxylic acids with alcohols or amines leading to the corresponding esters or amides, respectively [15-19]. Benziodazolone compounds 2, nitrogen analogs of benziodoxolones, are also known and offer the possibility of fine-tuning the reactivity by modifying the substituent on the nitrogen atom [20-23]. Thus, a number of reagents of 2 with various nitrogen-containing ligands and functional groups have been developed [24-28]. For example, benziodazolone compounds 2 supported by various ligands are known as atom-transfer reagents and can act as very effective electrophilic reagents for a variety of substrates, including azidation [20], alkynylation [24], and trifluorothiomethylation reagents [25]. Very recently, Zhang and coworkers investigated the synthesis and structural characterization of a stable fluorobenziodazolone compound and demonstrated that the novel reagent can efficiently perform ring-extended fluorination reactions for various three-membered ring compounds [26]. In addition, benziodazolones 3 are also capable of working as oxidants in dehydrogenative coupling reactions between various two-component molecules [27]. To the best of our knowledge, however, this is the first report where benziodazolones serve as the coupling assistant reagents, and their ligands serve as the coupling partners for alcohols and amines. Our group previously reported that bicyclic benziodazolone 4 could be employed as an efficient reagent for oxidatively assisted coupling of carboxylic 
acids with alcohols or amines in the presence of phosphines [28]. Therefore, in view of the growing interest in cyclic hypervalent iodine reagents, we focused on benzoidazolone 3 , whose acyloxy ligand is also a potential coupling partner for alcohols and amines.

(a) benziodoxoles - atom-transfer reagents and oxidants<smiles>[X]c1ccccc1C(=O)OI</smiles>

$\mathrm{X}=\mathrm{N}_{3}, \mathrm{CF}_{3}, \mathrm{NHR}, \mathrm{CN}$, alkynyl, OTf, OTs, etc.

(b) benziodazoles - atom-transfer reagents and oxidants<smiles>[R1]n1c(=O)c2ccccc2n1[Y]</smiles>

2<smiles></smiles>

3
$\mathrm{X}=\mathrm{N}_{3}$, alkynyl, $\mathrm{SCF}_{3}, \mathrm{~F}$

$\mathrm{R}=\mathrm{H}, \mathrm{COCH}_{3}$, Ts, etc
$\mathrm{R}_{2}=\mathrm{H}$, alkyl,

$\mathrm{CH}_{2} \mathrm{CO}_{2} \mathrm{CH}_{3}$, etc (c) bicyclic benziodazole - reagent for oxidatively assisted esterification and amidation<smiles>CC(C)N1C(=O)I2c3c(cccc31)C(=O)N2C(C)C</smiles>

(d) benziodazoles - reagents for aroylation of alcohols and amines<smiles>[R15]c1ccc(OC(=O)CCCCC)c(C(=O)N([R2])C)c1</smiles>

this work

Figure 1. Important iodine(III)-containing heterocycles, benziodoxolones and benziodazolones. (a): benziodoxoles-atom transfer reagents and oxidants; (b): bicyclic benziodazole-reagent for oxidatively assisted esterification and amidation; (c): benziodazoles-atom-transfer reagent and oxidants; (d): benziodazoles-reagent for aroylation of alcohols and amides; 1-benziodoxoles; 2-bicyclic benziodazole; 3, 4-beniodazoles; 5-benziodazoles.

In the present paper, we report a convenient and one-step procedure for the preparation of various 3-chlorobenzoyloxy-substituted benziodazolone derivatives 5 from the respective benzamides. In combination with $\mathrm{PPh}_{3}$ and pyridine, these new benziodazolones can smoothly react with alcohols or amines producing the corresponding esters or amides of 3-chlorobenzoic acid, respectively.

\section{Results and Discussion}

A novel series of 3-chlorobenzoyloxy-substituted benziodazolone derivatives $\mathbf{5 a}-\mathbf{j}$ were prepared in one step by the reaction of readily available benzamides 6 with $\mathrm{m}$ chloroperoxybenzoic acid ( $m$ CPBA) in acetonitrile at room temperature. In this method, by evaporation of the solvent and simply washing with ether, analytically pure benziodazolones 5 were obtained as stable, white solids in moderate to good yields. The structures of the products of $\mathbf{5}$ were confirmed by NMR spectroscopy, high-resolution mass spectrometry, and single-crystal X-ray crystallography of benziodazolone 5a (Scheme 1 and Figure 2). 


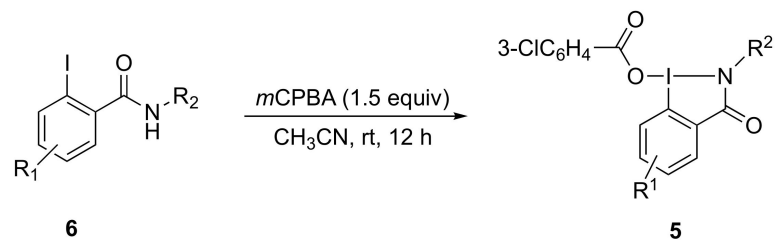<smiles>CC(C)N1C(=O)c2ccccc2I1OC(=O)c1cccc(Cl)c1</smiles>

5a (67\%)<smiles>O=C(OI1C(=O)c2ccccc2I1N1CCCCC1)c1cccc(Cl)c1</smiles>

5d $(51 \%)$<smiles>CCN1C(=O)c2ccccc2I1C(=O)Oc1cccc(Cl)c1</smiles>

5b (44\%)<smiles>O=C(OI1C(=O)c2ccccc2N1c1ccccc1)c1cccc(Cl)c1</smiles>

5e (76\%)<smiles></smiles>

5c $(77 \%)$<smiles>CC(C)N1C(=O)c2cc([N+](=O)[O-])ccc2I1OC(=O)c1cccc(Cl)c1</smiles>

5f $(56 \%)$<smiles>Cc1ccc2c(c1)I(OC(=O)c1cccc(Cl)c1)N(C(C)C)C2=O</smiles>

5g (53\%)<smiles>CC(C)N1C(=O)c2cccc([N+](=O)[O-])c2I1OC(=O)c1cccc(Cl)c1</smiles>

5h $(36 \%)$<smiles>CC(C)N(C)C(=O)c1cc(Br)ccc1OC(=O)c1cccc(Cl)c1</smiles>

$5 \mathbf{i}(71 \%)$<smiles>CC(C)N(C)C(=O)c1cc2ccccc2cc1OC(=O)c1cccc(Cl)c1</smiles>

5j (46\%)

Scheme 1. Preparation of benziodazolones 5. 5-benziodazoles; 6-iodobenzamides.

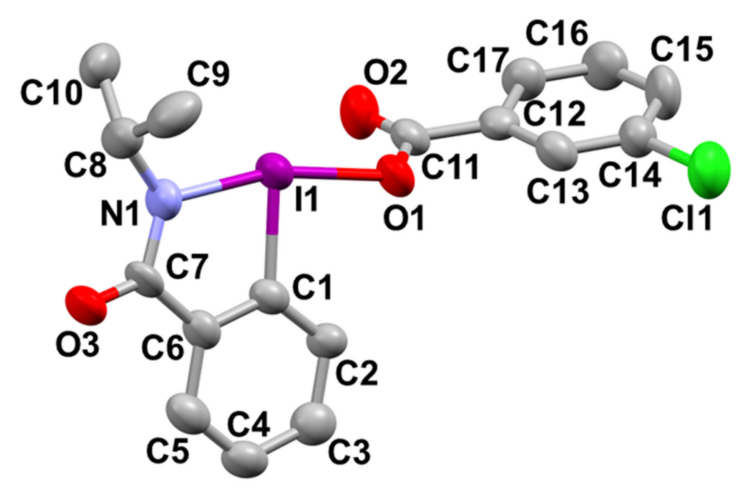

Figure 2. X-ray crystal structure of compound 5a. Typical bond distances in $\AA$ : I1-N1 = 2.126(5), $\mathrm{I} 1-\mathrm{C} 1=2.085(5), \mathrm{I} 1-\mathrm{O} 1=2.174(4) . \mathrm{C} 1-\mathrm{C} 17$; carbon atom, O1-O3; oxygen atom; Cl1; chlorine atom; I1; iodine atom.

Next, we investigated the use of benziodazolone 5a as a reagent for oxidatively assisted cross-coupling of alcohols with specially added carboxylic acids in the presence of a base (4-dimethylaminopyridine, DMAP) and $\mathrm{PPh}_{3}$ based on the previously reported procedures [15-19,27]. However, in contrast to previously reported reactions, yields of the desired carboxylic esters were low, and the main isolated products were $m$-chlorobenzoates formed by direct aroylation of alcohols with reagent 5a. Therefore, considering the importance of substituted benzoates in organic chemistry, we focused on the reactions of the 3-chlorobenzoyloxy ligand in benziodazolone 5 a with alcohols and amines.

Optimization studies of this new reaction using alcohol 7a as a model substrate have been performed in the absence of solvent with varying bases, phosphines, and ratios of reactants (Table 1$)$. The reaction of $\mathbf{7 a}$ in the presence of excessive benziodazolone $5 \mathbf{a}(2.4$ 
equiv.), $\mathrm{Ph}_{3} \mathrm{P}$ (2.4 equiv.) and DMAP (2.4 equiv.) afforded ester 8a in a $71 \%$ yield (entry 1 ). The further addition of $m$-chlorobenzoic acid to the reaction mixture did not improve the yield (entry 2). However, lowering the amounts of $\mathbf{5 a}$, DMAP, and $\mathrm{Ph}_{3} \mathrm{P}$ to 1.8 equivalents did not significantly change the yield (entry 3 ), and when the amounts of 5a, DMAP, and $\mathrm{Ph}_{3} \mathrm{P}$ were lowered to 1.2 equivalents, the yield increased to $84 \%$ (entry 4 ). Whereas, the further reduction of the amount of DMAP ( 0.6 equiv.) resulted in a significantly reduced yield (entry 5). Then, we tested several other bases (entries 6-11), and pyridine showed superior results with yields up to $91 \%$ (entry 9). When $\mathrm{Bu}_{3} \mathrm{P}$ was used instead of $\mathrm{Ph}_{3} \mathrm{P}$, the yield of the product was lower (entry 11). In addition, no product was formed in the absence of a phosphine (entry 12), and a low yield was observed in the absence of a base (entry 13). Next, we investigated the reactivity of the prepared benziodazolones 5 under the similar condition of entry 9 (entries 14-22). Likely due to the decomposition of the reagents during the reaction with $\mathbf{5 b}, \mathbf{c}, \mathbf{e}$, and $\mathbf{j}$, the reaction did not proceed efficiently, and $7 \mathbf{a}$ was detected in the reaction mixture (entries $14,15,17$, and 22). In contrast, when other benziodazolones $\mathbf{5 d}, \mathbf{f}-\mathbf{i}$, were employed, the reactions proceeded effectively to give the desired ester compound $\mathbf{8 a}$ in moderate to good yields (entries 16, 18-21).

Table 1. Optimization of the condensation of $n$-pentanol 7 a using benziodazolone 5 conditions ${ }^{1}$.

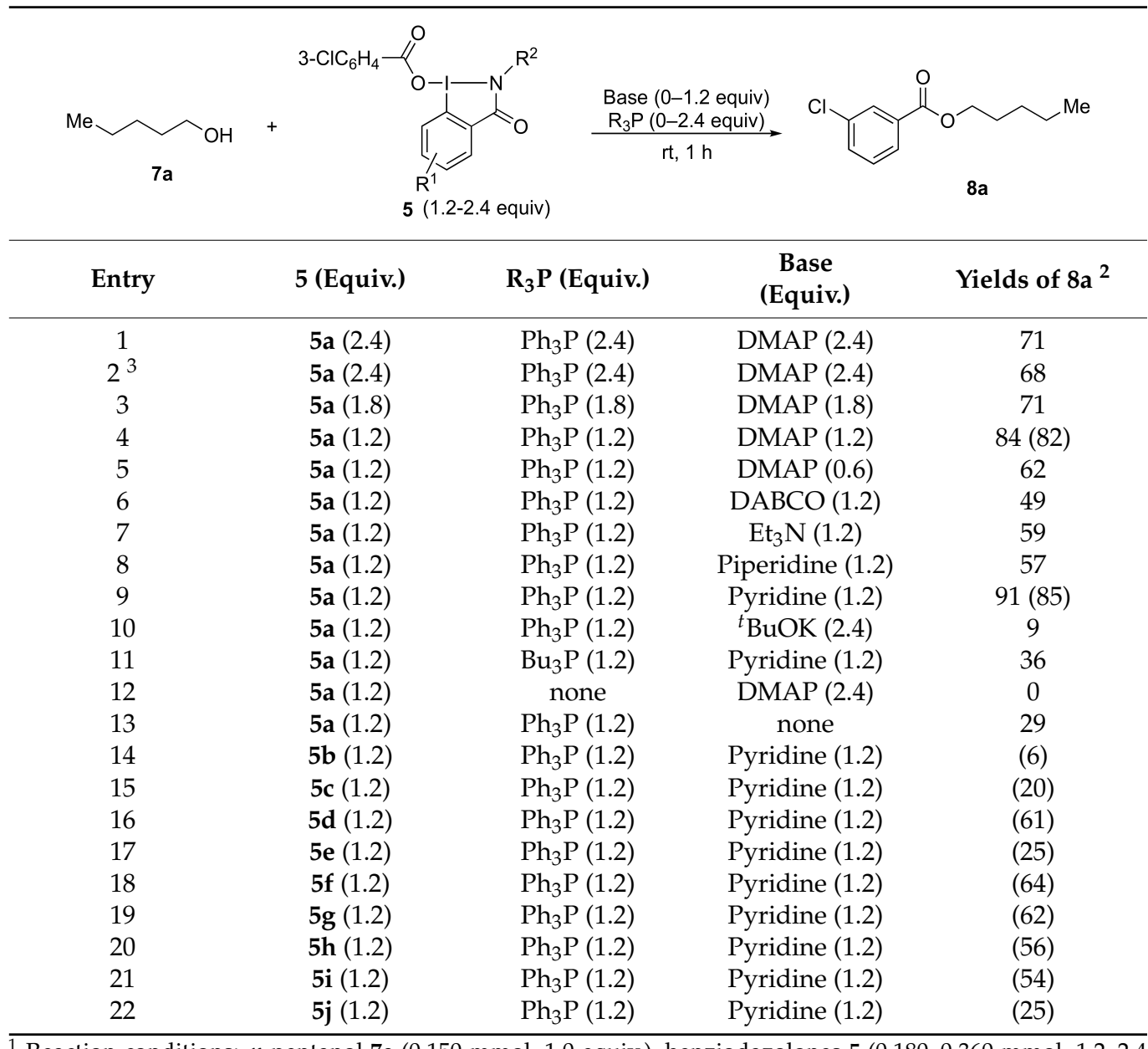

${ }^{1}$ Reaction conditions: $n$-pentanol 7a (0.150 mmol, 1.0 equiv.), benziodazolones 5 (0.180-0.360 mmol, 1.2-2.4 equiv.), phosphine ( $0-0.360 \mathrm{mmol}, 0-2.4$ equiv.), and base ( $0-0.360 \mathrm{mmol}, 0-2.4$ equiv.) stirred at room temperature under solvent-free conditions for $1 \mathrm{~h} .{ }^{2}$ Yields of product 8 a determined from ${ }^{1} \mathrm{H}$ NMR spectra of the reaction mixture (using 1,1,2,2-tetrachloroethane as an internal standard) are shown (numbers in parentheses show an isolated yield of 8 a). ${ }^{3} \mathrm{~m}$-Chlorobenzoic acid (1.4 equiv.) was added to the reaction mixture; $7 \mathbf{a}-n$-pentanol; 5-benziodazolones; 8a-pentyl 3-chlorobenzoate.

Using optimized reaction conditions, we investigated the scope of the esterification reaction of alcohols 7 with benziodazolone $5 \mathbf{a}$ (Scheme 2). In general, the reactions of 
primary and secondary alcohols $\mathbf{7} \mathbf{a}-\mathbf{h}$ afforded esters $\mathbf{8} \mathbf{a}-\mathbf{h}$ in moderate to high yields. In the reactions with alcohols $7 \mathbf{i}-1$ having unsaturated bonds, the respective ester compounds $8 \mathbf{i}-\mathbf{k}$ were obtained in low to good yields without any loss of unsaturated bonds, albeit with a low yield of 81 . Meanwhile, the reaction with sterically hindered tert-butanol gave only trace amounts of product $8 \mathrm{~m}$.

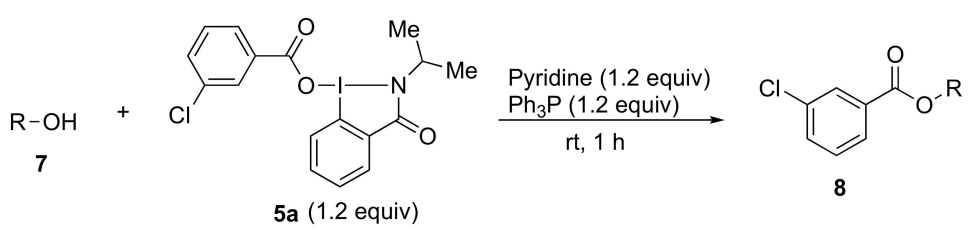<smiles>CCCCCOC(=O)c1cccc(Cl)c1</smiles>

8a (85\%)<smiles>CC(C)OC(=O)c1cccc(Cl)c1</smiles>

$8 f(54 \%)$<smiles>CCOC(=O)c1cccc(Cl)c1</smiles>

8b $(47 \%)$<smiles>COC(=O)c1cccc(Cl)c1</smiles>

8c $(70 \%)$<smiles>CCOC(=O)c1cccc(Cl)c1</smiles>

8d (76\%)<smiles>O=C(CCCCCc1ccccc1)c1cccc(Cl)c1</smiles>

8 e $(51 \%)$<smiles>CC(C)OC(=O)c1cccc(Cl)c1</smiles>

$8 g(61 \%)$<smiles>O=C(OC1CCCCC1)c1cccc(Cl)c1</smiles>

8h (56\%)<smiles>Cc1ccc(OC(=O)c2cccc(Cl)c2)cc1</smiles>

$8 \mathbf{i}(89 \%)$<smiles>C=CCOC(=O)c1cccc(Cl)c1</smiles>

8j (56\%)<smiles>C#CCCCOC(=O)c1cccc(Cl)c1</smiles>

8k $(71 \%)$<smiles>O=C(CCCc1cccnc1)c1cccc(Cl)c1</smiles>

$8 \mathbf{1}(18 \%)$<smiles>CC(C)(C)OC(=O)c1cccc(Cl)c1</smiles>

$8 \mathrm{~m}$ (trace)

Scheme 2. Esterification of alcohols using reagent 5a; 7-alcohols; 5a-benziodazolone; $\mathbf{8 a - m}$-esters.

Under similar reaction conditions, primary amines $\mathbf{9 a}-\mathbf{d}$ reacted with reagent $\mathbf{5 a}$ to form amides 10a-d in moderate to high yields (Scheme 3). In the case of the reaction with sterically hindered tert-butylamine, the desired amide compound $10 \mathrm{~d}$ was obtained in $43 \%$. This is likely because of the high nucleophilicity of amines.<smiles>[R]NC[NH3+]</smiles>

(1.2 equiv)<smiles>O=C(NCc1ccccc1)c1cccc(Cl)c1</smiles>

$10 \mathrm{a}(86 \%)$<smiles>CC(C)NC(=O)c1cccc(Cl)c1</smiles>

$10 \mathrm{~b}(87 \%)$

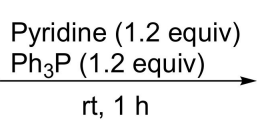

$\mathrm{rt}, 1 \mathrm{~h}$<smiles>O=C(NC1CCCCC1)c1cccc(Cl)c1</smiles>

$10 \mathrm{c}(65 \%)$<smiles>[R]NC(=O)c1cccc(Cl)c1</smiles>

10<smiles>CC(C)(C)NC(=O)c1cccc(Cl)c1</smiles>

10d (43\%)

Scheme 3. Amidation of amines using reagent 5a; 9-amines; 5a-benziodazolone; 10a-d-amides.

In the next study, the reactivity between benziodazolone 5 a and benziodoxolone 11, which could be easily synthesized from acetoxybenziodoxolone and 3-chlorobenzoic acid via a ligand exchange procedure, was compared. The condensation reaction of alcohol $7 \mathbf{a}$ and the prepared reagent $\mathbf{1 1}$ gave the desired ester 8a in only a 21\% yield (Scheme 4 ). This is because $\mathbf{1 1}$ was hardly miscible in the mixture. From this result, it was found that the efficiency of benziodazolone $\mathbf{5 a}$ was better than that of benziodoxolone $\mathbf{1 1}$ in this esterification reaction system. 


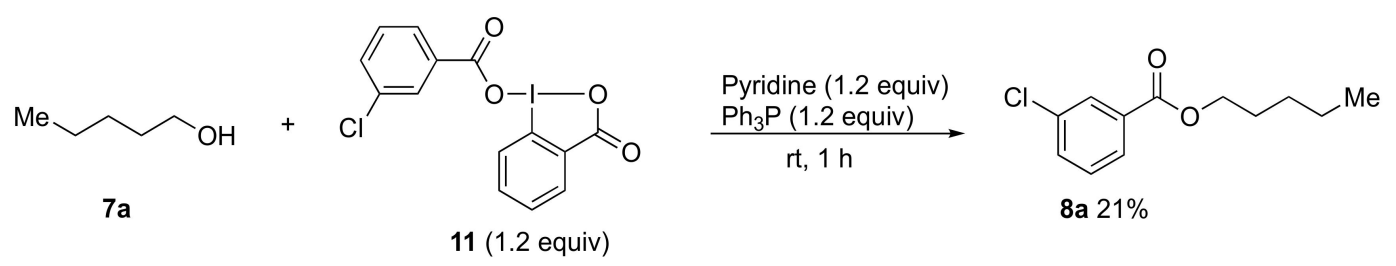

Scheme 4. Esterification of pentanol 7a using reagent 11; 7a- $n$-pentanol; 11-benziodoxolone; 8a-pentyl 3-chlorobenzoate.

In order to clarify the reaction mechanism of esterification, we carried out several control experiments (Scheme 5). Firstly, when the reaction of alcohol 7a with benziodazolone $5 \mathbf{a}$ did not proceed with the ligand exchange reaction, $5 \mathbf{a}$ was recovered from the reaction mixture in a quantitative amount (reaction 1). Then, we performed mass spectrometry experiments (see Supplementary Materials for detail). When 5a was treated with pyridine, the peak of the ligand exchange product 12 was not detected, but when $5 \mathbf{a}$ was treated with DMAP, the peak of the ligand exchange product 13 was detected. This may be due to DMAP being a stronger nucleophile than pyridine (reactions 2 and 3) [29]. Next, when the reaction of benziodazolone $5 \mathbf{a}$ with alcohol $\mathbf{7 a}$ and $\mathrm{Ph}_{3} \mathrm{P}$ was attempted, the peaks of $\mathrm{Ph}_{3} \mathrm{PO}$ and the estimated benziodazolone derivative structures such as $\mathbf{1 4}$ and $\mathbf{1 5}$ could be detected, while unfortunately, the mass peak of the expected ligand exchange intermediate 16 could not be observed directly (reaction 4 and Figure 3). The observed peaks were probably generated by the reaction of intermediate 16 from the ligand exchange reaction between $\mathrm{Ph}_{3} \mathrm{P}$ and $5 \mathrm{a}$ with moisture in the air during the mass experiment. Thus, these results may indicate that benziodazolone 5a reacts with $\mathrm{Ph}_{3} \mathrm{P}$ before pyridine or alcohol 7a. Notably, the reaction using benziodazolone $5 \mathrm{a}$ in the absence of $\mathrm{Ph}_{3} \mathrm{P}$ has been found to not proceed with the desired esterification at all (Table 1, entry 12).

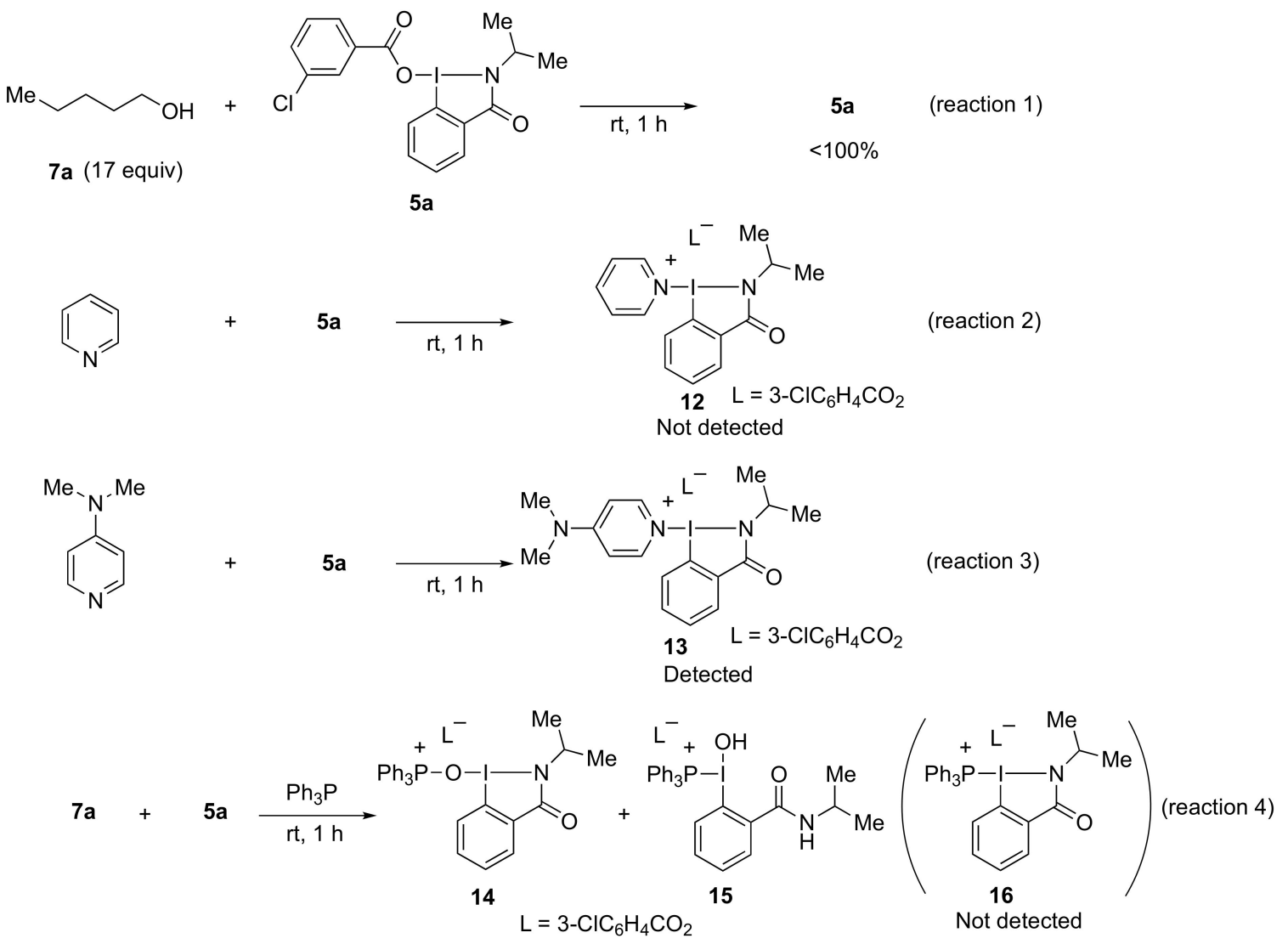

Scheme 5. Control experiment; 5a-benziodazolone; 7a- $n$-pentanol; 12,13, and 16-ligand exchange products; 14,15-estimated benziodazolone derivative structures. 


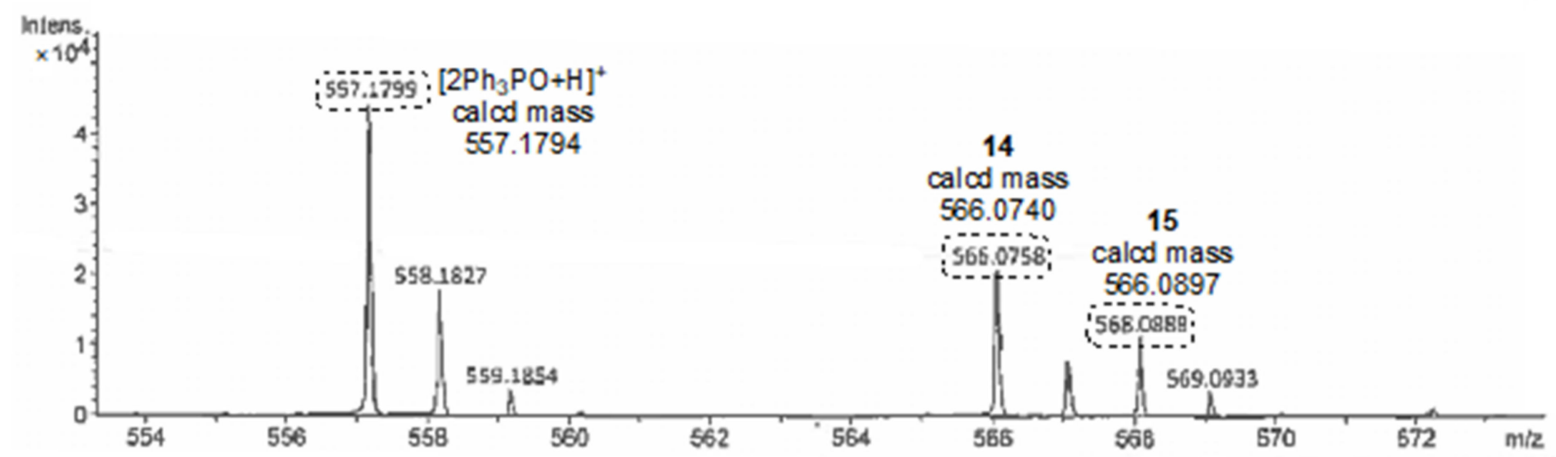

Figure 3. Mass result for Scheme 5, reaction 4; 14,15-estimated benziodazolone derivative structures.

Based on the results of these blank experiments and considering previously published mechanistic rationalizations $[15,17,19]$, we propose the following mechanistic scheme for the esterification reaction (Scheme 6). The reaction initially involves the ligand exchange between benziodazolone $5 \mathrm{a}$ and $\mathrm{Ph}_{3} \mathrm{P}$ via TS1 to generate the zwitterion intermediate 16. Then the carboxylate anion attacks the phosphorus center to produce intermediate 17. Next, $\mathbf{1 7}$ is converted to the phosphonium salt $\mathbf{1 8}$, which then reacts with pyridine and alcohol 7, respectively, to afford $N$-acyl pyridinium salt 19. Finally, 19 undergoes nucleophilic acyl substitution from the alkoxide of the counterion giving the desired ester 8. Although benziodoxolone requires a base such as DMAP in the ligand exchange with phosphine, it can smoothly proceed without a base in the case of benziodazolone 5a due to the significantly greater trans influence of the benziodazolone ring compared to the benziodoxolone ring [30]. Therefore, pyridine may mainly play a role in accelerating the nucleophilic acyl substitution for the formation of $N$-acyl pyridinium salts 19 .<smiles>CC(C)N1C(=O)c2ccccc2I1OC(=O)[Mg]</smiles>
$\mathrm{Ar}=3-\mathrm{ClC}_{6} \mathrm{H}_{4}$

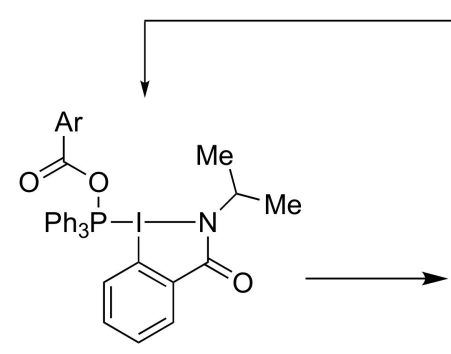

17<smiles>CC(C)NC(=O)c1ccccc1I</smiles>

18

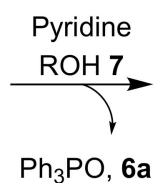<smiles>O=C([Al])[n+]1ccccc1</smiles>

19

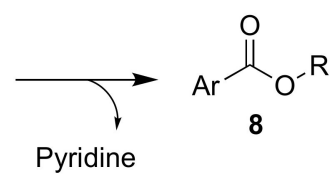

Pyridine

Scheme 6. Proposed mechanism of esterification reaction using reagent 5a; 5a-benziodazolone; $6 \mathbf{a}$-iodobezamide; Ts1-transition state 1; 15-estimated benziodazolone structure; 16,17-intermediates; 18-phosphonium salt; 19- $N$-acyl pyridinium salt.

\section{Materials and Methods}

\subsection{General Experimental Remarks}

All reactions were performed in open air with a stopper and oven-dried glassware. All commercial reagents were ACS grade and were used without further purification. NMR 
spectra were recorded on a Varian Inova $500 \mathrm{MHz}$ NMR spectrophotometer $\left({ }^{1} \mathrm{H}\right.$ NMR and ${ }^{13} \mathrm{C}$ NMR; Palo Alto, CA, USA) and Bruker $400 \mathrm{MHz}$ NMR spectrophotometer $\left({ }^{1} \mathrm{H}\right.$ NMR and ${ }^{13} \mathrm{C}$ NMR; Billerica, MA, USA). Melting points were determined in an open capillary tube with a Mel-temp II melting point apparatus. Infrared spectra were recorded on PerkinElmer Spectrum 1600 series FT-IR spectrometer (Waltham, MA, USA).

\subsection{General Procedure for Preparation of Iodobenzamide 6}

Amine was added dropwise at $0{ }^{\circ} \mathrm{C}$ to a stirred mixture of iodobenzoyl chloride and triethylamine in dichloromethane. The reaction was stirred for $1 \mathrm{~h}$ at $0{ }^{\circ} \mathrm{C}$. After completion of the reaction, water $(10-20 \mathrm{~mL})$ was added, and the mixture was extracted with dichloromethane. The organic phase was dried over anhydrous $\mathrm{Na}_{2} \mathrm{SO}_{4}$ and concentrated under reduced pressure. Purification by recrystallization $\left(\mathrm{CH}_{2} \mathrm{Cl}_{2}\right.$-hexane) afforded the analytically pure iodobenzamide 6 .

2-Iodo- $N$-isopropylbenzamide 6a [31]. The reaction with 2-iodobenzoyl chloride (3171 mg, $11.9 \mathrm{mmol})$, triethylamine $(2649 \mathrm{mg}, 26.2 \mathrm{mmol})$, and isopropylamine $(774 \mathrm{mg}, 13.1 \mathrm{mmol})$ in dichloromethane $(15 \mathrm{~mL})$ according to the general procedure afforded $3325 \mathrm{mg}(97 \%)$ of product 6a, isolated as a white solid: $\mathrm{mp} 136.4-137.7^{\circ} \mathrm{C}$ (lit. [31], mp 135-136 ${ }^{\circ} \mathrm{C}$ ); ${ }^{1} \mathrm{H}$ NMR $\left(500 \mathrm{MHz}, \mathrm{CDCl}_{3}\right): \delta 7.84(\mathrm{~d}, J=8.0 \mathrm{~Hz}, 1 \mathrm{H}), 7.41-7.34(\mathrm{~m}, 2 \mathrm{H}), 7.11-7.05(\mathrm{~m}, 1 \mathrm{H}), 5.57$ (brs, $1 \mathrm{H}), 4.38-4.22(\mathrm{~m}, 1 \mathrm{H})$, and $1.29(\mathrm{~d}, J=7.0 \mathrm{~Hz}, 1 \mathrm{H})$.

$\mathrm{N}$-Ethyl-2-iodobenzamide $6 \mathbf{b}$ [32]. The reaction with 2-iodobenzoyl chloride (1062 $\mathrm{mg}$, $3.99 \mathrm{mmol})$, triethylamine $(887 \mathrm{mg}, 8.77 \mathrm{mmol})$, and ethylamine $(198 \mathrm{mg}, 4.38 \mathrm{mmol})$ in dichloromethane $(15 \mathrm{~mL})$ according to the general procedure afforded $1032 \mathrm{mg}(94 \%)$ of product $6 \mathbf{b}$, isolated as a white solid: $\mathrm{mp} 114.3-115.5^{\circ} \mathrm{C}$ (lit. [32], 114-116 ${ }^{\circ} \mathrm{C}$ ); ${ }^{1} \mathrm{H}$ NMR $\left(500 \mathrm{MHz}, \mathrm{CDCl}_{3}\right): \delta 7.85(\mathrm{~d}, J=8.0 \mathrm{~Hz}, 1 \mathrm{H}), 7.41-7.33(\mathrm{~m}, 2 \mathrm{H}), 7.11-7.05(\mathrm{~m}, 1 \mathrm{H}), 5.78$ (brs, $1 \mathrm{H}), 3.62-3.40(\mathrm{~m}, 2 \mathrm{H})$, and $1.27(\mathrm{t}, J=7.3 \mathrm{~Hz}, 3 \mathrm{H})$.

$\mathrm{N}$-(tert-Butyl)-2-iodobenzamide 6c [33]. The reaction with 2-iodobenzoyl chloride (1063 mg, $3.99 \mathrm{mmol})$, triethylamine ( $888 \mathrm{mg}, 8.78 \mathrm{mmol})$, and tert-butylamine (321 mg, $4.39 \mathrm{mmol})$ in dichloromethane $(15 \mathrm{~mL})$ according to the general procedure afforded $1140 \mathrm{mg}(94 \%)$ of product 6c, isolated as a white solid: $\mathrm{mp} 122.2-124.1^{\circ} \mathrm{C}$ (Lit. [33], 120-122 $\left.{ }^{\circ} \mathrm{C}\right) ;{ }^{1} \mathrm{H}$ NMR $\left(500 \mathrm{MHz}, \mathrm{CDCl}_{3}\right): \delta 7.83(\mathrm{~d}, J=8.0 \mathrm{~Hz}, 1 \mathrm{H}), 7.41-7.32(\mathrm{~m}, 2 \mathrm{H}), 7.09-7.03(\mathrm{~m}, 1 \mathrm{H}), 5.55$ (brs, $1 \mathrm{H})$, and $1.49(\mathrm{~s}, 9 \mathrm{H})$.

$\mathrm{N}$-Cyclohexyl-2-iodobenzamide $6 \mathrm{~d}$ [33]. The reaction with 2-iodobenzoyl chloride (1025 $\mathrm{mg}$, $3.85 \mathrm{mmol})$, triethylamine ( $857 \mathrm{mg}, 8.46 \mathrm{mmol})$, and cyclohexylamine (420 mg, $4.23 \mathrm{mmol})$ in dichloromethane $(15 \mathrm{~mL})$ according to the general procedure afforded $1201 \mathrm{mg}(95 \%)$ of product $6 \mathrm{~d}$, isolated as a white solid: $\mathrm{mp} 141.2-142.3^{\circ} \mathrm{C}$ (lit. [33], $\mathrm{mp} 141-143^{\circ} \mathrm{C}$ ); ${ }^{1} \mathrm{H}$ NMR $\left(500 \mathrm{MHz}, \mathrm{CDCl}_{3}\right): \delta 7.85(\mathrm{~d}, J=8.0 \mathrm{~Hz}, 1 \mathrm{H}), 7.41-7.34(\mathrm{~m}, 2 \mathrm{H}), 7.11-7.05(\mathrm{~m}, 1 \mathrm{H}), 5.62$ (brs, $1 \mathrm{H}), 4.07-3.94(\mathrm{~m}, 1 \mathrm{H}), 2.12-2.04(\mathrm{~m}, 2 \mathrm{H}), 1.80-1.72(\mathrm{~m}, 2 \mathrm{H}), 1.69-1.61(\mathrm{~m}, 1 \mathrm{H}), 1.49-1.38(\mathrm{~m}$, $2 \mathrm{H})$, and $1.32-1.16(\mathrm{~m}, 3 \mathrm{H})$.

2-Iodo- $N$-phenylbenzamide 6e [33]. The reaction with 2-iodobenzoyl chloride (1089 $\mathrm{mg}$, $4.87 \mathrm{mmol})$, triethylamine $(908 \mathrm{mg}, 9.00 \mathrm{mmol})$, and aniline $(419 \mathrm{mg}, 4.50 \mathrm{mmol})$ in dichloromethane $(15 \mathrm{~mL})$ according to the general procedure afforded $1085 \mathrm{mg}(82 \%)$ of product 6e, isolated as a white solid: $\mathrm{mp} 144.2-145.0^{\circ} \mathrm{C}$ (Lit. [33], 144-146 ${ }^{\circ} \mathrm{C}$ ); ${ }^{1} \mathrm{H}$ NMR $\left(500 \mathrm{MHz}, \mathrm{CDCl}_{3}\right): \delta 7.92(\mathrm{~d}, J=8.0 \mathrm{~Hz}, 1 \mathrm{H}), 7.64(\mathrm{~d}, J=8.5 \mathrm{~Hz}, 2 \mathrm{H}), 7.54(\mathrm{~d}, J=8.0 \mathrm{~Hz}, 1 \mathrm{H})$, 7.47-7.42 $(\mathrm{m}, 2 \mathrm{H}), 7.42-7.35(\mathrm{~m}, 2 \mathrm{H})$, and 7.21-7.13 $(\mathrm{m}, 2 \mathrm{H})$.

2-Iodo- $N$-isopropyl-5-methylbenzamide $6 f$ [34]. The reaction with 2-iodo-5-methylbenzoyl chloride $(2140 \mathrm{mg}, 7.63 \mathrm{mmol})$, triethylamine $(1698 \mathrm{mg}, 16.8 \mathrm{mmol})$, and isopropylamine (496 mg, $8.39 \mathrm{mmol})$ in dichloromethane $(10 \mathrm{~mL})$ according to the general procedure afforded $2220 \mathrm{mg}(96 \%)$ of product $5 f$, isolated as a white solid: $\mathrm{mp} 147.8-148.7^{\circ} \mathrm{C}$ (Lit. [34], $\left.147-149^{\circ} \mathrm{C}\right) ;{ }^{1} \mathrm{H}$ NMR $\left(500 \mathrm{MHz}, \mathrm{CDCl}_{3}\right): \delta 7.68(\mathrm{~d}, J=8.0 \mathrm{~Hz}, 1 \mathrm{H}), 7.20(\mathrm{~s}, 1 \mathrm{H}), 6.90(\mathrm{~d}$, $J=8.0 \mathrm{~Hz}, 1 \mathrm{H}), 5.62(\mathrm{brs}, 1 \mathrm{H}), 4.33-4.233(\mathrm{~m}, 1 \mathrm{H}), 2.30(\mathrm{~s}, 3 \mathrm{H})$, and $1.28(\mathrm{~d}, J=7.0 \mathrm{~Hz}, 6 \mathrm{H})$; HRMS (ESI-positive): calcd for $\mathrm{C}_{11} \mathrm{H}_{15} \mathrm{INO}([\mathrm{M}+\mathrm{H}])^{+}: 304.0198$, found: 304.0196 . 
2-Iodo- $\mathrm{N}$-isopropyl-4-methylbenzamide $6 \mathrm{~g}$. The reaction with 2-iodo-4-methylbenzoyl chloride $(1070 \mathrm{mg}, 3.81 \mathrm{mmol})$, triethylamine $(849 \mathrm{mg}, 8.38 \mathrm{mmol})$, and isopropylamine $(248 \mathrm{mg}, 4.19 \mathrm{mmol})$ in dichloromethane $(10 \mathrm{~mL})$ according to the general procedure afforded $1118 \mathrm{mg}(97 \%)$ of product $5 \mathrm{~g}$, isolated as a white solid: $\mathrm{mp} 117.0-119.0{ }^{\circ} \mathrm{C}$; IR (neat) $\mathrm{cm}^{-1}$ : 3305, 3062, 2973, 2927, 1874, 1637, 1598. 1531, 1484, 1464, 1450, 1389, 1369, 1351, $1329,1289,1264,1178,1127,1036,883,850,831,821,805,601$, and 570; ${ }^{1} \mathrm{H}$ NMR $(500 \mathrm{MHz}$, $\left.\mathrm{CDCl}_{3}\right): \delta 7.69(\mathrm{~s}, 1 \mathrm{H}), 7.27(\mathrm{~d}, J=8.0 \mathrm{~Hz}, 1 \mathrm{H}), 7.15(\mathrm{~d}, J=8.0 \mathrm{~Hz}, 1 \mathrm{H}), 5.61$ (brs, $\left.1 \mathrm{H}\right)$, 4.33-4.22 (m, 1H), $2.31(\mathrm{~s}, 3 \mathrm{H})$, and $1.27(\mathrm{~d}, J=7.0 \mathrm{~Hz}, 6 \mathrm{H}) ;{ }^{13} \mathrm{C}$ NMR $\left(125 \mathrm{MHz}, \mathrm{CDCl}_{3}\right): \delta$ 168.5, 141.4, 140.2, 139.5, 128.9, 128.1, 92.4, 42.2, 22.6, and 20.7; HRMS (ESI-positive): calcd for $\mathrm{C}_{11} \mathrm{H}_{15} \mathrm{INO}([\mathrm{M}+\mathrm{H}])^{+}: 304.0198$, found: 304.0193 .

2-Iodo- $\mathrm{N}$-isopropyl-3-methylbenzamide $6 \mathrm{~h}$. The reaction with 2-iodo-3-methylbenzoyl chloride (1063 mg, $3.79 \mathrm{mmol})$, triethylamine $(844 \mathrm{mg}, 8.34 \mathrm{mmol})$, and isopropylamine $(224 \mathrm{mg}, 4.17 \mathrm{mmol})$ in dichloromethane $(10 \mathrm{~mL})$ according to the general procedure afforded $1084 \mathrm{mg}(94 \%)$ of product $\mathbf{6 h}$, isolated as a white solid: $\mathrm{mp} 135.6-137.2{ }^{\circ} \mathrm{C}$; ; IR (neat) $\mathrm{cm}^{-1}: 3248,3069,2970,2937,2875,1657,1551,1459,1366,1352,1331,1303,1266$, $1204,1156,1129,1012,919,858,805,780,738,718$, and 685; ${ }^{1} \mathrm{H}$ NMR $\left(500 \mathrm{MHz}, \mathrm{CDCl}_{3}\right): \delta$ 7.33-7.19 (m, 2H), 7.16-7.05 (m, 1H), $5.50($ brs, $1 \mathrm{H}), 4.41-4.21(\mathrm{~m}, 1 \mathrm{H}), 2.45(\mathrm{~s}, 3 \mathrm{H})$, and 1.29 $(\mathrm{d}, J=6.5 \mathrm{~Hz}, 6 \mathrm{H}) ;{ }^{13} \mathrm{C} \mathrm{NMR}\left(125 \mathrm{MHz}, \mathrm{CDCl}_{3}\right): \delta 169.9,144.2,142.8,130.2,128.1,124.9$, 99.3, 42.1, 29.1, and 22.6; HRMS (ESI-positive): calcd for $\mathrm{C}_{11} \mathrm{H}_{15} \mathrm{INO}([\mathrm{M}+\mathrm{H}])^{+}: 304.0198$, found: 304.0190 .

5-Bromo-2-iodo- $N$-isopropylbenzamide 6 i. The reaction with 5-bromo-2-iodobenzoyl chloride (1317 mg, $3.81 \mathrm{mmol})$, triethylamine $(848 \mathrm{mg}, 8.38 \mathrm{mmol})$, and isopropylamine $(248 \mathrm{mg}, 4.19 \mathrm{mmol})$ in dichloromethane $(10 \mathrm{~mL})$ according to the general procedure afforded $1347 \mathrm{mg}(96 \%)$ of product $6 \mathbf{i}$, isolated as a white solid: $\mathrm{mp} 187.8-188.5^{\circ} \mathrm{C}$; IR (neat) $\mathrm{cm}^{-1}: 3255,3073,2971,1642,1541,1453,1083,1017,900,810$, and 727; ${ }^{1} \mathrm{H} \mathrm{NMR}$ $\left(500 \mathrm{MHz}, \mathrm{CDCl}_{3}\right): \delta 7.69(\mathrm{~d}, J=8.0 \mathrm{~Hz}, 1 \mathrm{H}), 7.52-7.48(\mathrm{~m}, 1 \mathrm{H}), 7.24-7.19(\mathrm{~m}, 1 \mathrm{H}), 5.58$ (brs, $1 \mathrm{H}), 4.36-4.16(\mathrm{~m}, 1 \mathrm{H})$, and $1.29(\mathrm{~d}, J=6.5 \mathrm{~Hz}, 6 \mathrm{H}) ;{ }^{13} \mathrm{C} \mathrm{NMR}\left(125 \mathrm{MHz}, \mathrm{CDCl}_{3}\right): \delta$ 167.0, 144.1, 141.1, 134.0, 131.2, 122.6, 90.4, 42.4, and 22.6; HRMS (APCI-positive): calcd for $\mathrm{C}_{10} \mathrm{H}_{12}{ }^{79} \mathrm{BrINO}([\mathrm{M}+\mathrm{H}])^{+}: 367.9147$, found: 367.9122 .

3-Iodo- $\mathrm{N}$-isopropyl-2-naphthamide $6 \mathbf{j}$. The reaction with 3-iodo-2-naphthoyl chloride $(158 \mathrm{mg}, 0.50 \mathrm{mmol})$, triethylamine $(111 \mathrm{mg}, 1.1 \mathrm{mmol})$, and isopropylamine $(32.5 \mathrm{mg}$, $0.55 \mathrm{mmol})$ in dichloromethane $(5.0 \mathrm{~mL})$ according to the general procedure afforded $105 \mathrm{mg}(62 \%)$ of product $6 \mathbf{j}$, isolated as a white solid: $\mathrm{mp} 191.6-193.3^{\circ} \mathrm{C}$; IR (neat) $\mathrm{cm}^{-1}$ : $3279,2970,1642,1541,1124,954,908,895,880,805$, and $743 ;{ }^{1} \mathrm{H}$ NMR $\left(500 \mathrm{MHz}, \mathrm{CDCl}_{3}\right)$ : $\delta 8.38(\mathrm{~s}, 1 \mathrm{H}), 7.86(\mathrm{~s}, 1 \mathrm{H}), 7.84-7.79(\mathrm{~m}, 1 \mathrm{H}), 7.75-7.71(\mathrm{~m}, 1 \mathrm{H}), 7.57-7.50(\mathrm{~m}, 2 \mathrm{H}), 5.68$ (brs, $1 \mathrm{H}), 4.49-4.28(\mathrm{~m}, 1 \mathrm{H})$, and $1.33(\mathrm{~d}, J=6.5 \mathrm{~Hz}, 6 \mathrm{H}) ;{ }^{13} \mathrm{C} \mathrm{NMR}\left(75 \mathrm{MHz}, \mathrm{CDCl}_{3}\right): \delta$ 168.5, 139.3, 139.0, 134.9, 131.9, 128.1, 127.8, 127.4, 127.3, 126.7, 88.8, 42.3, and 22.7; HRMS (ESI-positive): calcd for $\mathrm{C}_{14} \mathrm{H}_{15} \mathrm{INO}([\mathrm{M}+\mathrm{H}])^{+}: 340.0198$, found: 340.0188 .

\subsection{General Procedure for Preparation of 3-Chlorobenzoyloxybenziodazolone 5}

Iodobenzamide 6 was added at $0{ }^{\circ} \mathrm{C}$ to a stirred mixture of $m$-CPBA in acetonitrile. The reaction was stirred for $12 \mathrm{~h}$ at room temperature. After completion of the reaction, the solvent was removed under reduced pressure to give solid residue. Then diethyl ether was added to the solid residue to prepare the suspended solution, which was filtered, and dried in a vacuum to give the desired 3-chlorobenzoyloxybenziodazolone 5.

2-Isopropyl-3-oxo-2,3-dihydro-1H-1 $\lambda^{3}$-benzo[d][1,2]iodazol-1-yl 3-chlorobenzoate 5a. The reaction with 2-iodo- $N$-isopropylbenzamide $6 \mathbf{a}(289 \mathrm{mg}, 1.00 \mathrm{mmol})$ and $m$-CPBA ( $259 \mathrm{mg}$, $1.50 \mathrm{mmol})$ in acetonitrile $(10 \mathrm{~mL})$ according to the general procedure afforded $296 \mathrm{mg}(67 \%)$ of product 5a, isolated as a white solid: $\mathrm{mp} 142.0-142.8^{\circ} \mathrm{C}$; IR (neat) $\mathrm{cm}^{-1}$ : 3067, 2967, 2932, 2875, 1627, 1588, 1570, 1296, 1257, and 739; ${ }^{1} \mathrm{H} \mathrm{NMR}\left(500 \mathrm{MHz}, \mathrm{CDCl}_{3}\right): \delta 8.28(\mathrm{~d}, J=8.0 \mathrm{~Hz}$, $1 \mathrm{H}), 8.22(\mathrm{dd}, J=7.8,1.8 \mathrm{~Hz}, 1 \mathrm{H}), 8.04(\mathrm{~s}, 1 \mathrm{H}), 7.96(\mathrm{~d}, J=7.5 \mathrm{~Hz}, 1 \mathrm{H}), 7.88-7.80(\mathrm{~m}, 1 \mathrm{H})$, 7.76-7.69 (m, 1H), $7.53(\mathrm{~d}, J=8.0 \mathrm{~Hz}, 1 \mathrm{H}), 7.45-7.37(\mathrm{~m}, 1 \mathrm{H}), 4.52($ sept., $J=6.5 \mathrm{~Hz}, 1 \mathrm{H})$, and $1.47(\mathrm{~d}, J=6.5 \mathrm{~Hz}, 6 \mathrm{H}) ;{ }^{13} \mathrm{C} \mathrm{NMR}\left(125 \mathrm{MHz}, \mathrm{CDCl}_{3}\right): \delta 171.3,166.0,134.6,133.7,133.6,132.3$, 
131.8, 131.0, 130.0, 129.8, 129.6, 127.9, 116.6, 46.7, and 24.4; HRMS (APCI-positive): calcd for $\mathrm{C}_{17} \mathrm{H}_{16} \mathrm{ClINO}_{3}([\mathrm{M}+\mathrm{H}])^{+}:$443.9863, found: 443.9877 .

Single crystals of product 5a suitable for X-ray crystallographic analysis were obtained by slow crystallization from the acetonitrile solution. X-ray diffraction data for 5a were collected on Rigaku RAPID II Image Plate system using graphite-monochromated CuK $\alpha$ radiation $(\lambda=1.54187 \AA)$ at $173 \mathrm{~K}$. The structure was solved by Sir 2011 and refined on $\mathrm{F}^{2}$ using ShelXle. Crystal data for $5 \mathrm{a} \mathrm{C}_{17} \mathrm{H}_{15} \mathrm{ClINO}_{3}$ are as follows: monoclinic, space group $\mathrm{P} 2{ }_{1} / \mathrm{c}, \mathrm{a}=12.6913(3), \mathrm{b}=14.8285(4), \mathrm{c}=18.3997(13) \AA, \alpha=90^{\circ}, \beta=104.695(7)^{\circ}, \gamma=90^{\circ}$, $\mathrm{V}=3349.4(3) \AA^{3}, \mathrm{Z}=8,22,784$ reflections measured, and 5815 unique $(4560 \mathrm{I}>2 \sigma /(\mathrm{I}))$; $\mathrm{R}_{\text {int }}=0.0780, \mathrm{R}_{\text {sigma }}=0.0834, \mathrm{R}_{1}(\mathrm{I}>2 \sigma /(\mathrm{I}))=0.0595, \mathrm{R}_{1}=0.0696, \mathrm{wR}_{\mathrm{all}}=0.1700$, and $\mathrm{S}=1.086$; please see the cif for more detailed information: CCDC-2122170.

2-Ethyl-3-oxo-2,3-dihydro-1H-1 $\lambda^{3}$-benzo[ $\left.d\right][1,2]$ iodazol-1-yl 3-chlorobenzoate $5 \mathrm{~b}$. The reaction with $N$-ethyl-2-iodobenzamide $6 \mathbf{b}(550 \mathrm{mg}, 2.00 \mathrm{mmol})$ and $\mathrm{m}$-CPBA (518 $\mathrm{mg}$, $3.00 \mathrm{mmol})$ in acetonitrile $(15 \mathrm{~mL})$ according to the general procedure afforded $380 \mathrm{mg}$ (44\%) of product $5 \mathbf{b}$, isolated as a white solid: $\mathrm{mp} 76.0-78.0^{\circ} \mathrm{C}$; IR (neat) $\mathrm{cm}^{-1}$ : 3074, 2969, 2935, 2876, 1627, 1590, 1571, 1442, 1319, 1263, 756, and 739; $\left.{ }^{1} \mathrm{H} \mathrm{NMR} \mathrm{(500} \mathrm{MHz}^{\mathrm{CDCl}} \mathrm{CDC}_{3}\right)$ : $\delta 8.29(\mathrm{~d}, J=8.0 \mathrm{~Hz}, 1 \mathrm{H}), 8.23(\mathrm{~d}, J=7.5 \mathrm{~Hz}, 1 \mathrm{H}), 8.04(\mathrm{~s}, 1 \mathrm{H}), 7.96(\mathrm{~d}, J=8.0 \mathrm{~Hz}, 1 \mathrm{H})$, 7.90-7.83 (m, 1H), $7.73(\mathrm{t}, J=8.0 \mathrm{~Hz}, 1 \mathrm{H}), 7.53(\mathrm{~d}, J=8.0 \mathrm{~Hz}, 1 \mathrm{H}), 7.44-7.37(\mathrm{~m}, 1 \mathrm{H}), 3.76$ $(\mathrm{q}, J=7.3 \mathrm{~Hz}, 2 \mathrm{H})$, and $1.37(\mathrm{t}, J=7.3 \mathrm{~Hz}, 3 \mathrm{H}) ;{ }^{13} \mathrm{C} \mathrm{NMR}\left(125 \mathrm{MHz}, \mathrm{CDCl}_{3}\right): \delta 171.3$, $166.3,134.9,134.4,133.6,132.5,132.4,131.9,131.0,130.0,129.8,129.6,127.9,116.9,38.3$, and 16.2; HRMS (ESI-positive): calcd for $\mathrm{C}_{9} \mathrm{H}_{11} \mathrm{INO}_{2}\left(\left[\mathrm{M}-3 \mathrm{ClC}_{6} \mathrm{H}_{4} \mathrm{CO}_{2}+\mathrm{H}\right]\right)^{+}:$291.9834, found: 291.9817.

2-(tert-Butyl)-3-oxo-2,3-dihydro-1H-1 $\lambda^{3}$-benzo[ $\left.d\right][1,2]$ iodazol-1-yl 3-chlorobenzoate $5 c$. The reaction with $N$-(tert-butyl)-2-iodobenzamide $6 \mathrm{c}(606 \mathrm{mg}, 2.00 \mathrm{mmol})$ and $\mathrm{m}$-CPBA (518 mg, $3.00 \mathrm{mmol})$ in acetonitrile $(15 \mathrm{~mL})$ according to the general procedure afforded $705 \mathrm{mg}(77 \%)$ of product 5c, isolated as a white solid: $\mathrm{mp} 170.8-172.0^{\circ} \mathrm{C}$; IR (neat) $\mathrm{cm}^{-1}: 3070,2966,1628,1585$, $1570,1438,1317,1262,930,756$, and 740; ${ }^{1} \mathrm{H}$ NMR (500 MHz, $\left.\mathrm{CDCl}_{3}\right): \delta 8.26(\mathrm{~d}, J=8.5 \mathrm{~Hz}, 1 \mathrm{H})$, $8.15(\mathrm{~d}, J=7.5 \mathrm{~Hz}, 1 \mathrm{H}), 8.03(\mathrm{~s}, 1 \mathrm{H}), 7.95(\mathrm{~d}, J=7.5 \mathrm{~Hz}, 1 \mathrm{H}), 7.84(\mathrm{t}, J=7.5 \mathrm{~Hz}, 1 \mathrm{H}), 7.73-7.67$ $(\mathrm{m}, 1 \mathrm{H}), 7.52(\mathrm{~d}, J=7.5 \mathrm{~Hz}, 1 \mathrm{H}), 7.42-7.37(\mathrm{~m}, 1 \mathrm{H})$, and $1.71(\mathrm{~s}, 9 \mathrm{H}) ;{ }^{13} \mathrm{C} \mathrm{NMR}(125 \mathrm{MHz}$, $\left.\mathrm{CDCl}_{3}\right): \delta 171.3,165.8,135.0,134.5,134.4,133.7,132.3,131.8,130.9,129.6,129.5,127.9,114.8$, 58.2, and 30.2; HRMS (ESI-positive): calcd for $\mathrm{C}_{11} \mathrm{H}_{15} \mathrm{INO}_{2}\left(\left[\mathrm{M}-3 \mathrm{ClC}_{6} \mathrm{H}_{4} \mathrm{CO}_{2}+\mathrm{H}\right]\right)^{+}: 320.0147$, found: 320.0137.

2-Cyclohexyl-3-oxo-2,3-dihydro-1H-1 $\lambda^{3}$-benzo[d][1,2]iodazol-1-yl 3-chlorobenzoate 5d. The reaction with $N$-cyclohexyl-2-iodobenzamide $6 \mathbf{d}(750 \mathrm{mg}, 2.28 \mathrm{mmol})$ and $\mathrm{m}$-CPBA $(590 \mathrm{mg}, 3.42 \mathrm{mmol})$ in acetonitrile $(17.1 \mathrm{~mL})$ according to the general procedure afforded $633 \mathrm{mg}(51 \%)$ of product 5d, isolated as a white solid: $\mathrm{mp} 159.0-160.3^{\circ} \mathrm{C}$; IR (neat) $\mathrm{cm}^{-1}$ : $3071,2929,2854,1629,1589,1566,1439,1323,1294,1621,1067,967,756,739$, and $659 ;{ }^{1} \mathrm{H}$ $\operatorname{NMR}\left(500 \mathrm{MHz}, \mathrm{CDCl}_{3}\right): \delta 8.29(\mathrm{~d}, J=8.5 \mathrm{~Hz}, 1 \mathrm{H}), 8.22(\mathrm{~d}, J=7.8 \mathrm{~Hz}, 1 \mathrm{H}), 8.04(\mathrm{~s}, 1 \mathrm{H})$, $7.96(\mathrm{~d}, J=7.5 \mathrm{~Hz}, 1 \mathrm{H}), 7.83(\mathrm{t}, J=7.8 \mathrm{~Hz}, 1 \mathrm{H}), 7.75-7.69(\mathrm{~m}, 1 \mathrm{H}), 7.53(\mathrm{~d}, J=8.0 \mathrm{~Hz}, 1 \mathrm{H})$, 7.43-7.38 (m, 1H), 4.30-4.06 (m, 1H), 2.34-2.13 (m, 2H), 1.95-1.85 (m, 2H), 1.78-1.71 (m, 1H), $1.54-1.43(\mathrm{~m}, 4 \mathrm{H})$, and 1.30-1.20 (m, 1H); ${ }^{13} \mathrm{C} \mathrm{NMR}\left(125 \mathrm{MHz}, \mathrm{CDCl}_{3}\right): \delta 171.4,165.9,134.5$, 134.4, 133.7, 133.6, 132.3, 131.8, 131.0, 130.0, 129.8, 129.6, 127.8, 116.9, 54.2, 35.5, 25.7, and 25.3; HRMS (ESI-positive): calcd for $\mathrm{C}_{13} \mathrm{H}_{17} \mathrm{INO}_{2}\left(\left[\mathrm{M}-3 \mathrm{ClC}_{6} \mathrm{H}_{4} \mathrm{CO}_{2}+\mathrm{H}\right]\right)^{+}:$346.0304, found: 346.0286 .

3-Oxo-2-phenyl-2,3-dihydro-1H-1 $\lambda^{3}$-benzo[d][1,2]iodazol-1-yl 3-chlorobenzoate 5e. The reaction with 2-iodo- $N$-phenylbenzamide 6 e $(750 \mathrm{mg}, 2.32 \mathrm{mmol})$ and $\mathrm{m}$-CPBA (601 $\mathrm{mg}$, $3.48 \mathrm{mmol})$ in acetonitrile $(17.4 \mathrm{~mL})$ according to the general procedure afforded $841 \mathrm{mg}$ (76\%) of product 5e, isolated as a white solid: $\mathrm{mp} 154.8^{\circ} \mathrm{C}$ (decomp.); IR (neat) $\mathrm{cm}^{-1}$ : 3067, 3033, 1637, 1586, 1569, 1488, 1441, 1507, 1262, 1125, 754, 741, and 659; ${ }^{1} \mathrm{H} \mathrm{NMR} \mathrm{(500} \mathrm{MHz,}$ $\left.\mathrm{CDCl}_{3}\right): \delta 8.34(\mathrm{~d}, J=6.0 \mathrm{~Hz}, 1 \mathrm{H}), 8.27(\mathrm{~d}, J=8.5 \mathrm{~Hz}, 1 \mathrm{H}), 8.03(\mathrm{~s}, 1 \mathrm{H}), 7.97-7.90(\mathrm{~m}, 2 \mathrm{H})$, 7.81-7.74 (m, 1H), $7.54(\mathrm{~d}, J=8.0 \mathrm{~Hz}, 1 \mathrm{H}), 7.50-7.39(\mathrm{~m}, 5 \mathrm{H})$, and $7.35-7.28(\mathrm{~m}, 1 \mathrm{H}) ;{ }^{13} \mathrm{C}$ NMR $\left(125 \mathrm{MHz}, \mathrm{CDCl}_{3}\right)$ : $\delta 170.9,164.6,138.1,135.3,134.5,132.9,132.8,132.6,132.6,131.2$, 
129.9, 129.8, 129.7, 129.7, 128.0, 127.2, 126.4, and 117.2; HRMS (ESI-positive): calcd for $\mathrm{C}_{13} \mathrm{H}_{11} \mathrm{INO}_{2}\left(\left[\mathrm{M}-3 \mathrm{ClC}_{6} \mathrm{H}_{4} \mathrm{CO}_{2}+\mathrm{H}\right]\right)^{+}:$339.9834, found: 339.9807 .

2-Isopropyl-5-methyl-3-oxo-2,3-dihydro-1H-1 $\lambda^{3}$-benzo[ $\left.d\right][1,2]$ iodazol-1-yl 3-chlorobenzoate 5f. The reaction with 2-iodo- $N$-isopropyl-5-methylbenzamide $6 f(606 \mathrm{mg}, 2.00 \mathrm{mmol}$ ) and $m$-CPBA $(518 \mathrm{mg}, 3.00 \mathrm{mmol})$ in acetonitrile $(15 \mathrm{~mL})$ according to the general procedure afforded $513 \mathrm{mg}(56 \%)$ of product $5 \mathrm{f}$, isolated as a white solid: $\mathrm{mp} 148.0-15.0{ }^{\circ} \mathrm{C}$; IR (neat) $\mathrm{cm}^{-1}$ : 3067, 2965, 2927, 2874, 1630, 1574, 1456, 1307, 1260, 1143, 756, and 740; ${ }^{1} \mathrm{H}$ NMR (500 MHz, $\left.\mathrm{CDCl}_{3}\right): \delta 8.11(\mathrm{~d}, J=9.5 \mathrm{~Hz}, 1 \mathrm{H}), 8.02(\mathrm{~s}, 2 \mathrm{H}), 7.95(\mathrm{~d}, J=7.5 \mathrm{~Hz}, 1 \mathrm{H}), 7.66-7.61(\mathrm{~m}, 1 \mathrm{H})$, 7.54-7.50 (m, 1H), 7.43-7.37 (m, 1H), 4.61-4.42 (m, 1H), $2.54(\mathrm{~s}, 3 \mathrm{H})$, and $1.45(\mathrm{~d}, J=6.5 \mathrm{~Hz}$, $6 \mathrm{H}) ;{ }^{13} \mathrm{C} \mathrm{NMR}\left(125 \mathrm{MHz}, \mathrm{CDCl}_{3}\right): \delta 171.2,166.1,141.9,135.6,134.3,133.8,133.4,132.3,132.2$, $129.8,129.6,129.6,127.9,112.8,46.7,24.3$, and 20.9; HRMS (ESI-positive): calcd for $\mathrm{C}_{11} \mathrm{H}_{15} \mathrm{INO}_{2}$ $\left(\left[\mathrm{M}-3 \mathrm{ClC}_{6} \mathrm{H}_{4} \mathrm{CO}_{2}+\mathrm{H}\right]\right)^{+}: 320.0147$, found: 320.0141 .

2-Isopropyl-6-methyl-3-oxo-2,3-dihydro-1H-1 $\lambda^{3}$-benzo[ $\left.d\right][1,2]$ iodazol-1-yl 3-chlorobenzoate $5 \mathrm{~g}$. The reaction with 2-iodo- $N$-isopropyl-4-methylbenzamide $6 \mathrm{~g}(606 \mathrm{mg}, 2.00 \mathrm{mmol})$ and $m$-CPBA $(518 \mathrm{mg}, 3.00 \mathrm{mmol})$ in acetonitrile $(15 \mathrm{~mL})$ according to the general procedure afforded $486 \mathrm{mg}$ (53\%) of product $6 \mathrm{~g}$, isolated as a white solid: $\mathrm{mp} 150.2-151.5^{\circ} \mathrm{C}$; IR (neat) $\mathrm{cm}^{-1}: 3073$, 2964, 2929, 1618, 1569, 1463, 1313, 1295, 1260, 1143, 757, 740, and 667; ${ }^{1} \mathrm{H}$ NMR (500 MHz, $\left.\mathrm{CDCl}_{3}\right): \delta 8.07(\mathrm{~d}, J=7.5 \mathrm{~Hz}, 1 \mathrm{H}), 8.06-8.02(\mathrm{~m}, 2 \mathrm{H}), 7.95(\mathrm{~d}, J=7.8 \mathrm{~Hz}, 1 \mathrm{H}), 7.55-7.49(\mathrm{~m}$, $2 \mathrm{H}), 7.41(\mathrm{t}, J=7.8 \mathrm{~Hz}, 1 \mathrm{H}), 4.60-4.41(\mathrm{~m}, 1 \mathrm{H}), 2.56(\mathrm{~s}, 3 \mathrm{H})$, and $1.45(\mathrm{~d}, J=6.5 \mathrm{~Hz}, 6 \mathrm{H}) ;{ }^{13} \mathrm{C}$ $\mathrm{NMR}\left(125 \mathrm{MHz}_{2} \mathrm{CDCl}_{3}\right)$ : $\delta 171.3,166.1,146.0,134.4,133.8,132.3,132.1,131.5,131.0,129.9$, $129.8,129.6,127.8,116.8,46.6,24.4$, and 22.3; HRMS (ESI-positive): calcd for $\mathrm{C}_{11} \mathrm{H}_{15} \mathrm{INO}_{2}$ $\left(\left[\mathrm{M}-3 \mathrm{ClC}_{6} \mathrm{H}_{4} \mathrm{CO}_{2}+\mathrm{H}\right]\right)^{+}: 320.0147$, found: 320.0140 .

2-Isopropyl-7-methyl-3-oxo-2,3-dihydro- $1 H-1 \lambda^{3}$-benzo[ $\left.d\right][1,2]$ iodazol-1-yl 3-chlorobenzoate $5 \mathrm{~h}$. The reaction with 2-iodo- $N$-isopropyl-3-methylbenzamide $6 \mathbf{h}(736 \mathrm{mg}, 2.00 \mathrm{mmol})$ and $m$-CPBA $(518 \mathrm{mg}, 1.50 \mathrm{mmol})$ in acetonitrile $(15 \mathrm{~mL})$ according to the general procedure afforded $738 \mathrm{mg}(71 \%)$ of product $5 \mathrm{~h}$, isolated as a white solid: $\mathrm{mp} 117.2-117.8^{\circ} \mathrm{C}$; IR (KBr) cm ${ }^{-1}$ : 3127 , 2960, 2909, 1648, 1608, 1569, 1384, 1317, 1139, and 745; ${ }^{1} \mathrm{H} \mathrm{NMR}\left(400 \mathrm{MHz}, \mathrm{CDCl}_{3}\right)$ : $\delta 8.08-8.02$ $(\mathrm{m}, 1 \mathrm{H}), 7.97(\mathrm{~s}, 1 \mathrm{H}), 7.89(\mathrm{~d}, J=8.4 \mathrm{~Hz}, 2 \mathrm{H}), 7.64-7.56(\mathrm{~m}, 2 \mathrm{H}), 7.49(\mathrm{t}, J=8.0 \mathrm{~Hz}, 1 \mathrm{H}), 7.47(\mathrm{t}$, $J=7.8 \mathrm{~Hz}, 1 \mathrm{H}), 4.57-4.44(\mathrm{~m}, 1 \mathrm{H}), 2.80(\mathrm{~s}, 3 \mathrm{H})$, and $1.49(\mathrm{~d}, J=6.4 \mathrm{~Hz}, 6 \mathrm{H}) ;{ }^{13} \mathrm{C} \mathrm{NMR}(100 \mathrm{MHz}$, $\left.\mathrm{CDCl}_{3}\right): \delta 172.1,166.7,140.2,138.2,134.9,134.4,134.4,132.1,130.9,129.8,129.6,127.7,118.9,47.1$, 24.4, and 23.6; HRMS (ESI-positive): calcd for $\mathrm{C}_{11} \mathrm{H}_{15} \mathrm{INO}_{2}\left(\left[\mathrm{M}-3 \mathrm{ClC}_{6} \mathrm{H}_{4} \mathrm{CO}_{2}+\mathrm{H}\right]\right)^{+}: 320.0147$, found: 320.0146 .

5-Bromo-2-isopropyl-3-oxo-2,3-dihydro-1H-1 $\lambda^{3}$-benzo[d][1,2]iodazol-1-yl 3-chlorobenzoate 5i. The reaction with 5-bromo-2-iodo- $N$-isopropylbenzamide $6 \mathbf{i}(736 \mathrm{mg}, 2.00 \mathrm{mmol})$ and $\mathrm{m}$ CPBA $(518 \mathrm{mg}, 1.50 \mathrm{mmol})$ in acetonitrile $(15 \mathrm{~mL})$ according to the general procedure afforded $738 \mathrm{mg}(71 \%)$ of product $5 \mathbf{i}$, isolated as a white solid: $\mathrm{mp} 151.6-152.6{ }^{\circ} \mathrm{C}$; IR (neat) $\mathrm{cm}^{-1}$ : 3103, 3066, 2968, 2931, 1636, 1617, 1568, 1557, 1464, 1443, 1405, 1305, 1260, 1141, 1070, 959, 903, 813, 755, and 742; ${ }^{1} \mathrm{H}$ NMR (500 MHz, $\left.\mathrm{CDCl}_{3}\right): \delta 8.35-8.33(\mathrm{~m}, 1 \mathrm{H}), 8.13(\mathrm{~d}, J=9.0 \mathrm{~Hz}, 2 \mathrm{H}), 8.02-8.00$ $(\mathrm{m}, 1 \mathrm{H}), 7.95-7.91(\mathrm{~m}, 2 \mathrm{H}), 7.56-7.52(\mathrm{~m}, 1 \mathrm{H}), 7.43-7.39(\mathrm{~m}, 1 \mathrm{H}), 4.56-4.41(\mathrm{~m}, 1 \mathrm{H})$, and 1.46 $(\mathrm{d}, J=6.5 \mathrm{~Hz}, 6 \mathrm{H}) ;{ }^{13} \mathrm{C} \mathrm{NMR}\left(125 \mathrm{MHz}, \mathrm{CDCl}_{3}\right): \delta 171.3,164.5,137.4,135.4,134.7,134.4,133.3$, 132.5, 131.4, 129.8, 129.7, 127.8, 126.4, 114.5, 47.0, and 24.3; HRMS (ESI-positive): calcd for $\mathrm{C}_{10} \mathrm{H}_{12}{ }^{79} \mathrm{BrINO}_{2}\left(\left[\mathrm{M}-3 \mathrm{ClC}_{6} \mathrm{H}_{4} \mathrm{CO}_{2}+\mathrm{H}\right]\right)^{+}$: 383.9096, found: 383.9101 .

2-Isopropyl-3-oxo-2,3-dihydro-1H-1 $\lambda^{3}$-naphtho[2,3-d][1,2]iodazol-1-yl 3-chlorobenzoate 5j. The reaction with 3-iodo- $N$-isopropyl-2-naphthamide $6 \mathbf{j}(85 \mathrm{mg}, 0.250 \mathrm{mmol}$ ) and $\mathrm{m}$ CPBA $(65 \mathrm{mg}, 0.375 \mathrm{mmol})$ in acetonitrile $(1.9 \mathrm{~mL})$ according to the general procedure afforded $56 \mathrm{mg}(46 \%)$ of product $5 \mathbf{j}$, isolated as a white solid: $\mathrm{mp} 153.3-155.0^{\circ} \mathrm{C}$; IR (neat) $\mathrm{cm}^{-1}$ : 3058, 2966, 2922, 1636, 1619, 1569, 1296, 1144, 758, and 740; ${ }^{1} \mathrm{H}$ NMR $(500 \mathrm{MHz}$, $\left.\mathrm{CDCl}_{3}\right): \delta 8.74(\mathrm{~s}, 2 \mathrm{H}), 8.14-8.08(\mathrm{~m}, 2 \mathrm{H}), 8.04-7.98(\mathrm{~m}, 2 \mathrm{H}), 7.77-7.69(\mathrm{~m}, 2 \mathrm{H}), 7.58-7.53$ $(\mathrm{m}, 1 \mathrm{H}), 7.44(\mathrm{t}, J=7.8 \mathrm{~Hz}, 1 \mathrm{H}), 4.66-4.47(\mathrm{~m}, 1 \mathrm{H})$, and $1.49(\mathrm{~d}, J=6.5 \mathrm{~Hz}, 6 \mathrm{H}) ;{ }^{13} \mathrm{C}$ $\mathrm{NMR}\left(75 \mathrm{MHz}_{1} \mathrm{CDCl}_{3}\right)$ : $\delta 171.3,165.9,136.7,134.4,133.8,133.4,132.4,130.6,129.9,129.7$, 129.3, 129.2, 128.9, 128.7, 128.3, 127.9, 111.1, 46.7, and 24.2; HRMS (ESI-positive): calcd for $\mathrm{C}_{14} \mathrm{H}_{14} \mathrm{INO}_{2}\left(\left[\mathrm{M}-3 \mathrm{ClC}_{6} \mathrm{H}_{4} \mathrm{CO}_{2}+\mathrm{H}\right]\right)^{+}:$356.0147, found: 356.0119 . 


\subsection{Preparation of 3-Chlorobenzoyloxybenziodoxole $\mathbf{1 1}$}

A mixture of 3,3-dimethyl-1 $\lambda^{3}$-benzo[d][1,2]iodaoxol-1(3H)-yl acetate (1530 mg, $5.00 \mathrm{mmol}$ ) in $\mathrm{CHCl}_{3}(15 \mathrm{~mL})$ was added under stirring. The reaction was stirred at reflux for $18 \mathrm{~h}$. After completion of the reaction, the solvent was removed under reduced pressure to give solid residue. The solid residue was filtrated and washed with diethyl ether and hexane several times and then dried under a vacuum to give $1712 \mathrm{mg}(85 \%)$ of product 11, isolated as white solid; IR $\left(\mathrm{KBr} \mathrm{cm}^{-1} 3059,1695,1623,1569,1309,1266,1235,1116\right.$, and $747 ;{ }^{1} \mathrm{H}$ NMR $\left(400 \mathrm{MHz}, \mathrm{CDCl}_{3}\right)$ : $\delta 8.30(\mathrm{~d}, J=7.6 \mathrm{~Hz}, 1 \mathrm{H}), 8.13-7.95(\mathrm{~m}, 4 \mathrm{H}), 7.81-7.73(\mathrm{~m}, 1 \mathrm{H}), 7.65-7.57(\mathrm{~m}, 1 \mathrm{H})$, and $7.45(\mathrm{~d}$, $J=8.0 \mathrm{~Hz}, 1 \mathrm{H}) ;{ }^{13} \mathrm{C} \mathrm{NMR}\left(100 \mathrm{MHz}, \mathrm{CDCl}_{3}\right): \delta 170.1,168.0,136.5,134.8,133.5,131.5,130.7$, 130.1, 130.0, 129.1, 128.8, 128.3, and 118.7; HRMS (ESI-positive): calcd for $\mathrm{C}_{14} \mathrm{H}_{8}{ }^{35} \mathrm{ClIO}_{4}$ ([M + $\mathrm{H}])^{+}: 402.9229$, found: 402.9214 .

\subsection{Esterification of 1-Pentanol with 3-Cholorbenzoyloxybenziodazolones}

Pentyl 3-chlorobenzoate 8a [35]. Triphenylphosphine (47 mg, $0.180 \mathrm{mmol})$, pyridine (14 mg, $0.180 \mathrm{mmol})$, and 1-pentanol $7 \mathrm{a}(13 \mathrm{mg}, 0.150 \mathrm{mmol})$ were added to a test tube containing benziodazolones $5(0.180 \mathrm{mmol})$. The mixture was then stirred at room temperature for $1 \mathrm{~h}$. After the reaction was completed, dichloromethane $(3.0 \mathrm{~mL})$ was used to transfer the reaction mixture to a separatory funnel. Then saturated $\mathrm{NaHCO}_{3}(3.0 \mathrm{~mL})$ was added, and the reaction mixture was extracted with dichloromethane. The organic layer was dried over anhydrous $\mathrm{Na}_{2} \mathrm{SO}_{4}$ and concentrated under reduced pressure. $\mathrm{Pu}$ rification by preparative TLC (hexane:ethyl acetate $=3: 1$ ) afforded the analytically pentyl 3-chlorobenzoate 8a, isolated as a colorless oil; IR (neat) $\mathrm{cm}^{-1}$ : 2961, 1724, 1576, 1469, 1293, 1256, 1127, 967, and 675; ${ }^{1} \mathrm{H} \mathrm{NMR}\left(500 \mathrm{MHz}, \mathrm{CDCl}_{3}\right): \delta 8.01(\mathrm{~s}, 1 \mathrm{H}), 7.93(\mathrm{~d}, J=7.8 \mathrm{~Hz}, 1 \mathrm{H})$, $7.54-7.49(\mathrm{~m}, 1 \mathrm{H}), 7.38(\mathrm{~d}, J=7.8 \mathrm{~Hz}, 1 \mathrm{H}), 4.32(\mathrm{t}, J=6.9 \mathrm{~Hz}, 2 \mathrm{H}), 1.77$ (quint, $J=6.9 \mathrm{~Hz}$, $1 \mathrm{H}), 1.48-1.43(\mathrm{~m}, 4 \mathrm{H})$, and $0.93(\mathrm{t}, J=7.3 \mathrm{~Hz}, 3 \mathrm{H}) ;{ }^{13} \mathrm{C} \mathrm{NMR}\left(75 \mathrm{MHz}, \mathrm{CDCl}_{3}\right): \delta 165.4$, 134.5, 132.8, 132.3, 129.7, 129.6, 127.7, 65.6, 28.4, 28.2, 22.4, and 14.0; HRMS (APCI-positive): calcd for $\mathrm{C}_{12} \mathrm{H}_{17} \mathrm{ClO}_{2}([\mathrm{M}+\mathrm{H}])^{+}:$227.0839, found: 227.0846.

The reaction of 1-pentanol $7 \mathrm{a}(13 \mathrm{mg}, 0.150 \mathrm{mmol})$ using benziodazolone $5 \mathrm{a}(80 \mathrm{mg}$, $0.180 \mathrm{mmol}$ ) according to the general procedure afforded $29 \mathrm{mg}(85 \%)$ of product $8 \mathbf{a}$, isolated as a colorless oil.

The reaction of 1-pentanol 7a (13 mg, $0.150 \mathrm{mmol})$ using benziodazolone $5 \mathbf{b}(99 \mathrm{mg}$, $0.180 \mathrm{mmol}$ ) according to the general procedure afforded $2 \mathrm{mg}(6 \%)$ of product $8 \mathrm{a}$, isolated as a colorless oil.

The reaction of 1-pentanol $7 \mathrm{a}(13 \mathrm{mg}, 0.150 \mathrm{mmol})$ using benziodazolone $5 \mathrm{c}(82 \mathrm{mg}$, $0.180 \mathrm{mmol}$ ) according to the general procedure afforded $3 \mathrm{mg}(10 \%)$ of product $8 \mathbf{a}$, isolated as a colorless oil.

The reaction of 1-pentanol $7 \mathbf{a}(13 \mathrm{mg}, 0.150 \mathrm{mmol})$ using benziodazolone $5 \mathbf{d}(88 \mathrm{mg}$, $0.180 \mathrm{mmol})$ according to the general procedure afforded $21 \mathrm{mg}(61 \%)$ of product $8 \mathrm{a}$, isolated as a colorless oil.

The reaction of 1-pentanol 7a $(13 \mathrm{mg}, 0.150 \mathrm{mmol})$ using benziodazolone $5 \mathbf{e}(86 \mathrm{mg}$, $0.180 \mathrm{mmol}$ ) according to the general procedure afforded $9 \mathrm{mg}(25 \%)$ of product $8 \mathrm{a}$, isolated as a colorless oil.

The reaction of 1-pentanol $7 \mathrm{a}(13 \mathrm{mg}, 0.150 \mathrm{mmol})$ using benziodazolone $5 \mathrm{f}(82 \mathrm{mg}$, $0.180 \mathrm{mmol})$ according to the general procedure afforded $22 \mathrm{mg}(64 \%)$ of product $8 \mathrm{a}$, isolated as a colorless oil.

The reaction of 1-pentanol 7a (13 mg, $0.150 \mathrm{mmol})$ using benziodazolone $5 \mathrm{~g}(82 \mathrm{mg}$, $0.180 \mathrm{mmol})$ according to the general procedure afforded $21 \mathrm{mg}(62 \%)$ of product $8 \mathrm{a}$, isolated as a colorless oil.

The reaction of 1-pentanol 7a $(13 \mathrm{mg}, 0.150 \mathrm{mmol})$ using benziodazolone $5 \mathrm{~h}(82 \mathrm{mg}$, $0.180 \mathrm{mmol}$ ) according to the general procedure afforded $19 \mathrm{mg}(56 \%)$ of product 8a, isolated as a colorless oil. 
The reaction of 1-pentanol 7a $(13 \mathrm{mg}, 0.150 \mathrm{mmol})$ using benziodazolone $5 \mathbf{i}$ (94 $\mathrm{mg}$, $0.180 \mathrm{mmol}$ ) according to the general procedure afforded $19 \mathrm{mg}$ (54\%) of product 8a, isolated as a colorless oil.

The reaction of 1-pentanol 7a $(5.1 \mathrm{mg}, 0.057 \mathrm{mmol})$ using benziodazolone $5 \mathbf{j}$ (34 $\mathrm{mg}$, $0.069 \mathrm{mmol})$ according to the general procedure afforded $3.2 \mathrm{mg}(25 \%)$ of product $8 \mathbf{a}$, isolated as a colorless oil.

\subsection{Typical Procedure for Esterification and Amidation Using Benziodazolones}

Triphenylphosphine (47 mg, $0.18 \mathrm{mmol})$, pyridine (14 mg, $0.18 \mathrm{mmol})$, and alcohol 7 $(0.15 \mathrm{mmol})$ or amine $9(0.15 \mathrm{mmol})$ were added to a test tube containing benziodazolones 5 $(0.180 \mathrm{mmol})$. The mixture was then stirred at room temperature for $1 \mathrm{~h}$. After the reaction was completed, dichloromethane $(3.0 \mathrm{~mL})$ was used to transfer the reaction mixture to a separatory funnel. Then saturated $\mathrm{NaHCO}_{3}(3.0 \mathrm{~mL})$ was added, and the reaction mixture was extracted with dichloromethane. The organic layer was dried over anhydrous $\mathrm{Na}_{2} \mathrm{SO}_{4}$ and concentrated under reduced pressure. Purification by preparative TLC (hexane:ethyl acetate $=3: 1$ ) afforded the analytically pure 8 or $\mathbf{1 0}$.

Ethyl 3-chlorobenzoate $\mathbf{8 b}$ [36]. The reaction of ethanol $\mathbf{7 b}(7 \mathrm{mg}, 0.150 \mathrm{mmol})$ according to the general procedure afforded $13 \mathrm{mg}(47 \%)$ of product $8 \mathbf{b}$, isolated as a colorless oil; IR (neat) $\mathrm{cm}^{-1}: 2924,1727,1573,1466,1370,1293,1281,1256,915$, and $749 ;{ }^{1} \mathrm{H} \mathrm{NMR}(500 \mathrm{MHz}$, $\left.\mathrm{CDCl}_{3}\right): \delta 8.03(\mathrm{~s}, 1 \mathrm{H}), 7.94(\mathrm{~d}, J=7.5 \mathrm{~Hz}, 1 \mathrm{H}), 7.56-7.52(\mathrm{~m}, 1 \mathrm{H}), 7.39(\mathrm{t}, J=7.5 \mathrm{~Hz}, 1 \mathrm{H}), 4.39$ $(\mathrm{q}, J=7.0 \mathrm{~Hz}, 2 \mathrm{H})$, and $1.41(\mathrm{t}, J=7.0 \mathrm{~Hz}, 3 \mathrm{H})$; HRMS (APCI-positive): calcd for $\mathrm{C}_{9} \mathrm{H}_{10} \mathrm{ClO}_{2}$ $([\mathrm{M}+\mathrm{H}])^{+}:$185.0369, found: 185.0389 .

Methyl 3-chlorobenzoate 8c [37]. The reaction of methanol 7c (5 mg, $0.150 \mathrm{mmol})$ according to the general procedure afforded $18 \mathrm{mg}(70 \%)$ of product $8 \mathrm{c}$, isolated as a colorless oil; ${ }^{1} \mathrm{H}$ NMR $\left(500 \mathrm{MHz}, \mathrm{CDCl}_{3}\right): \delta 8.02(\mathrm{~s}, 1 \mathrm{H}), 7.93(\mathrm{~d}, J=7.8 \mathrm{~Hz}, 1 \mathrm{H}), 7.56-7.51(\mathrm{~m}, 1 \mathrm{H}), 7.39$ $(\mathrm{t}, J=7.8 \mathrm{~Hz}, 1 \mathrm{H})$, and $3.93(\mathrm{~s}, 3 \mathrm{H}) ;{ }^{13} \mathrm{C} \mathrm{NMR}\left(125 \mathrm{MHz} \mathrm{CDCl}_{3}\right): \delta 165.9,134.5,133.0,131.9$, $130.0,129.7,127.7$, and 52.4 .

Benzyl 3-chlorobenzoate $8 d$ [38]. The reaction of benzyl alcohol 7d (16 $\mathrm{mg}, 0.150 \mathrm{mmol})$ according to the general procedure afforded $28 \mathrm{mg}(76 \%)$ of product $8 \mathrm{~d}$, isolated as a colorless oil; IR (neat) $\mathrm{cm}^{-1}: 2955,1721,1576,1429,1290,1278,1124,955$, and $659 ;{ }^{1} \mathrm{H}$ NMR $\left(500 \mathrm{MHz}, \mathrm{CDCl}_{3}\right): \delta 8.04(\mathrm{~s}, 1 \mathrm{H}), 7.95(\mathrm{~d}, J=8.0 \mathrm{~Hz}, 1 \mathrm{H}), 7.54-7.50(\mathrm{~m}, 1 \mathrm{H}), 7.47-7.42(\mathrm{~m}$, $2 \mathrm{H}), 7.42-7.33(\mathrm{~m}, 4 \mathrm{H})$, and $5.36(\mathrm{~s}, 2 \mathrm{H}) ;{ }^{13} \mathrm{C} \mathrm{NMR}\left(125 \mathrm{MHz}, \mathrm{CDCl}_{3}\right): \delta 165.2,135.6,134.5$, 133.1, 131.9, 129.7, 129.7, 128.7, 128.4, 128.3, 127.8, and 67.1; HRMS (ESI-positive): calcd for $\mathrm{C}_{14} \mathrm{H}_{11} \mathrm{ClO}_{2} \mathrm{Na}([\mathrm{M}+\mathrm{Na}])^{+}: 269.0345$, found: 269.0341 .

5-Phenylpentyl 3-chlorobenzoate 8e. The reaction of 5-phenyl-1-pentyl alcohol 7e (25 mg, $0.150 \mathrm{mmol}$ ) according to the general procedure afforded $23 \mathrm{mg}(51 \%)$ of product $8 \mathbf{e}$, isolated as a colorless oil; IR (neat) $\mathrm{cm}^{-1}$ : 2937, 1724, 1576, 1429, 1293, 1256, 1124, 958, 699, 675, and 659; ${ }^{1} \mathrm{H}$ NMR (500 MHz, $\left.\mathrm{CDCl}_{3}\right): \delta 7.99(\mathrm{~s}, 1 \mathrm{H}), 7.90(\mathrm{~d}, J=8.0 \mathrm{~Hz}, 1 \mathrm{H}), 7.51(\mathrm{~d}$, $J=8.0 \mathrm{~Hz}, 1 \mathrm{H}), 7.39-7.33(\mathrm{~m}, 1 \mathrm{H}), 7.30-7.22(\mathrm{~m}, 2 \mathrm{H}), 7.20-7.13(\mathrm{~m}, 3 \mathrm{H}), 4.31(\mathrm{t}, J=6.5 \mathrm{~Hz}$, $2 \mathrm{H}), 2.75-2.53(\mathrm{~m}, 2 \mathrm{H}), 1.83-1.75(\mathrm{~m}, 2 \mathrm{H}), 1.73-1.65(\mathrm{~m}, 2 \mathrm{H})$, and $1.52-1.43(\mathrm{~m}, 2 \mathrm{H}) ;{ }^{13} \mathrm{C}$ NMR $\left(125 \mathrm{MHz}, \mathrm{CDCl}_{3}\right)$ : $\delta$ 165.4, 142.3, 134.5, 132.8, 132.2, 129.7, 129.6, 128.4, 128.3, 127.7, $125.7,65.4,35.8,31.0,28.5$, and 25.6; HRMS (APCI-positive): calcd for $\mathrm{C}_{18} \mathrm{H}_{20} \mathrm{ClO}_{2}$ ([M + $\mathrm{H}])^{+}:$303.1152, found: 303.1173.

Isopropyl 3-chlorobenzoate $8 \mathrm{f}$ [39]. The reaction of isopropanol $\mathbf{7 f}$ ( $9 \mathrm{mg}, 0.150 \mathrm{mmol})$ according to the general procedure afforded $16 \mathrm{mg}(54 \%)$ of product $\mathbf{8 f}$, isolated as a colorless oil; ${ }^{1} \mathrm{H}$ NMR (500 MHz, $\left.\mathrm{CDCl}_{3}\right): \delta 8.01(\mathrm{~s}, 1 \mathrm{H}), 7.92(\mathrm{~d}, J=7.9 \mathrm{~Hz}, 1 \mathrm{H}), 7.54-7.50$ $(\mathrm{m}, 1 \mathrm{H}), 7.37(\mathrm{~d}, J=7.9 \mathrm{~Hz}, 1 \mathrm{H}), 5.25(\mathrm{sept}, J=6.5 \mathrm{~Hz}, 1 \mathrm{H})$, and $1.37(\mathrm{~d}, J=6.5 \mathrm{~Hz}, 6 \mathrm{H})$.

1-Phenylethyl 3-chlorobenzoate 8g [40]. The reaction of 1-phenylethyl alcohol $7 \mathbf{g}$ (18 mg, $0.150 \mathrm{mmol})$ according to the general procedure afforded $24 \mathrm{mg}(61 \%)$ of product $8 \mathrm{~g}$, isolated as a colorless oil; IR (neat) $\mathrm{cm}^{-1}: 2985,1723,1574,1496,1456,1428,1256,1129$, and 697; ${ }^{1} \mathrm{H}$ NMR $\left(500 \mathrm{MHz}, \mathrm{CDCl}_{3}\right): \delta 8.05(\mathrm{~s}, 1 \mathrm{H}), 7.97(\mathrm{~d}, J=8.0 \mathrm{~Hz}, 1 \mathrm{H}), 7.56-7.52(\mathrm{~m}, 1 \mathrm{H})$, $7.45(\mathrm{~d}, J=8.0 \mathrm{~Hz}, 1 \mathrm{H}), 7.42-7.36(\mathrm{~m}, 3 \mathrm{H}), 7.36-7.30(\mathrm{~m}, 1 \mathrm{H}), 6.14(\mathrm{q}, J=6.9 \mathrm{~Hz}, 1 \mathrm{H})$, and 
$1.69(\mathrm{~d}, J=6.9 \mathrm{~Hz}, 3 \mathrm{H}) ;{ }^{13} \mathrm{C}$ NMR $\left(125 \mathrm{MHz}, \mathrm{CDCl}_{3}\right): \delta 164.6,141.4,134.5,133.0,132.3,129.7$, 128.7, 128.1, 127.8, 126.1, 73.5, and 22.3; HRMS (ESI-positive): calcd for $\mathrm{C}_{15} \mathrm{H}_{13} \mathrm{ClO}_{2} \mathrm{Na}$ ([M $+\mathrm{Na}])^{+}: 283.0502$, found: 283.0489 .

Cyclohexyl 3-chlorobenzoate $8 \mathrm{~h}$ [40]. The reaction of cyclohexanol $7 \mathbf{h}$ (15 $\mathrm{mg}, 0.150 \mathrm{mmol})$ according to the general procedure afforded $20 \mathrm{mg}(56 \%)$ of product $8 \mathrm{~h}$, isolated as a colorless oil; IR (neat) $\mathrm{cm}^{-1}$ : 2937, 1721, 1576, 1469, 1290, 1253, 1124, 945, 749, and 678; ${ }^{1} \mathrm{H}$ NMR (500 MHz, $\left.\mathrm{CDCl}_{3}\right): \delta 8.03-8.00(\mathrm{~m}, 1 \mathrm{H}), 7.95-7.91(\mathrm{~m}, 1 \mathrm{H}), 7.54-7.50(\mathrm{~m}, 1 \mathrm{H}), 7.38(\mathrm{t}$, $J=8.0 \mathrm{~Hz}, 1 \mathrm{H}), 5.09-4.94(\mathrm{~m}, 1 \mathrm{H}), 1.99-1.91(\mathrm{~m}, 2 \mathrm{H}), 1.84-1.75(\mathrm{~m}, 2 \mathrm{H}), 1.64-1.53(\mathrm{~m}, 3 \mathrm{H})$, 1.50-1.40 (m, 2H), and 1.40-1.30 (m, 1H); $\left.{ }^{13} \mathrm{C} \mathrm{NMR} \mathrm{(125} \mathrm{MHz,} \mathrm{CDCl}_{3}\right): \delta 164.8,134.4,132.8$, 132.7, 129.6, 127.7, 73.7, 31.6, 25.4, and 23.7; HRMS (APCI-positive): calcd for $\mathrm{C}_{13} \mathrm{H}_{16} \mathrm{ClO}_{2}$ $([\mathrm{M}+\mathrm{H}])^{+}:$239.0839, found: 239.0843 .

$p$-Tolyl 3-chlorobenzoate $8 \mathbf{i}$ [41]. The reaction of $p$-cresol $7 \mathbf{i}$ (16 mg, $0.150 \mathrm{mmol}$ ) according to the general procedure afforded $33 \mathrm{mg}(89 \%)$ of product $\mathbf{8 i}$, isolated as a white solid, $\mathrm{mp}$ 75.4-76.0 ${ }^{\circ} \mathrm{C}$; IR (neat) $\mathrm{cm}^{-1}: 2925,1742,1578,1474,1288,1251,1197,1165,1106,811$, and $743 ;{ }^{1} \mathrm{H}$ NMR $\left(500 \mathrm{MHz}, \mathrm{CDCl}_{3}\right): \delta 8.17(\mathrm{~s}, 1 \mathrm{H}), 8.07(\mathrm{~d}, J=8.0 \mathrm{~Hz}, 1 \mathrm{H}), 7.61-7.56(\mathrm{~m}, 1 \mathrm{H})$, $7.43(\mathrm{t}, J=8.0 \mathrm{~Hz}, 1 \mathrm{H}), 7.22(\mathrm{~d}, J=8.5 \mathrm{~Hz}, 2 \mathrm{H}), 7.08(\mathrm{~d}, J=8.5 \mathrm{~Hz}, 2 \mathrm{H})$, and $2.37(\mathrm{~s}, 3 \mathrm{H}) ;{ }^{13} \mathrm{C}$ NMR (125 MHz, $\left.\mathrm{CDCl}_{3}\right)$ : $\delta$ 164.2, 148.5, 135.8, 134.7, 133.5, 131.5, 130.2, 130.1, 129.9, 128.3, 121.2, and 20.9; HRMS (ESI-positive): calcd for $\mathrm{C}_{14} \mathrm{H}_{12} \mathrm{ClO}_{2}([\mathrm{M}+\mathrm{H}])^{+}: 247.0526$, found: 247.0528 .

Allyl 3-chlorobenzoate $8 \mathbf{j}$ [42]. The reaction of allyl alcohol $7 \mathbf{j}$ (13 $\mathrm{mg}, 0.150 \mathrm{mmol}$ ) according to the general procedure afforded $16 \mathrm{mg}(56 \%)$ of product $\mathbf{8 j}$, isolated as a colorless oil; IR (neat) $\mathrm{cm}^{-1}$ : 3077, 2945, 2883, 1726, 1576, 1426, 1289, 1256, 1128, and 748; ${ }^{1} \mathrm{H} \mathrm{NMR}$ $\left(400 \mathrm{MHz}, \mathrm{CDCl}_{3}\right): \delta 8.07-8.02(\mathrm{~m}, 1 \mathrm{H}), 7.95(\mathrm{~d}, J=7.8 \mathrm{~Hz}, 1 \mathrm{H}), 7.60-7.50(\mathrm{~m}, 1 \mathrm{H}), 7.39(\mathrm{t}$, $J=7.8 \mathrm{~Hz}, 1 \mathrm{H}), 5.42(\mathrm{dd}, J=17.2,1.6 \mathrm{~Hz}, 1 \mathrm{H}), 5.31(\mathrm{dd}, J=10.4,1.6 \mathrm{~Hz}, 1 \mathrm{H})$, and $4.83(\mathrm{~d}$, $J=5.6 \mathrm{~Hz}, 2 \mathrm{H}) ;{ }^{13} \mathrm{C} \mathrm{NMR}\left(100 \mathrm{MHz}, \mathrm{CDCl}_{3}\right): \delta 165.1,134.6,133.0,131.9,129.7,127.8,118.7$, 74.2, and 66.0; HRMS (EI): calcd for $\mathrm{C}_{10} \mathrm{H}_{9}{ }^{35} \mathrm{ClO}_{2}([\mathrm{M}+\mathrm{H}])^{+}:$196.0291, found: 196.0285.

Hex-5-yn-1-yl 3-chlorobenzoate 8k. The reaction of 5-hexyn-1-ol 7k (15 mg, $0.150 \mathrm{mmol})$ according to the general procedure afforded $25 \mathrm{mg}(71 \%)$ of product $8 \mathbf{k}$, isolated as a colorless oil; IR (neat) cm $\mathrm{cm}^{-1}$ : 3298, 3074, 2951, 2870, 2117, 1722, 1575, 1428, 1285, 1260, 1130, 747, and 641; ${ }^{1} \mathrm{H}$ NMR $\left(400 \mathrm{MHz} \mathrm{CDCl}_{3}\right): \delta 8.01(\mathrm{~s}, 1 \mathrm{H}), 7.93(\mathrm{~d}, J=7.8 \mathrm{~Hz}, 1 \mathrm{H}), 7.56-7.50$ $(\mathrm{m}, 1 \mathrm{H}), 7.38(\mathrm{~d}, J=7.8 \mathrm{~Hz}, 1 \mathrm{H}), 4.36(\mathrm{t}, J=6.4 \mathrm{~Hz}, 2 \mathrm{H}), 2.36-2.16(\mathrm{~m}, 2 \mathrm{H}), 1.99(\mathrm{t}, J=2.8 \mathrm{~Hz}$, $1 \mathrm{H}), 1.96-1.87(\mathrm{~m}, 2 \mathrm{H}), 1.75-$ and $1.64(\mathrm{~m}, 2 \mathrm{H}) ;{ }^{13} \mathrm{C} \mathrm{NMR}\left(100 \mathrm{MHz}, \mathrm{CDCl}_{3}\right): \delta 165.4,134.5$, 132.9, 132.1, 129.7, 129.6, 127.7, 83.8, 68.9, 64.9, 27.7, 25.0, and 18.1; HRMS (CI): calcd for $\mathrm{C}_{13} \mathrm{H}_{13}{ }^{35} \mathrm{ClO}_{2}([\mathrm{M}+\mathrm{H}])^{+}:$254.0942, found:254.0934.

3-(Pyridin-3-yl)propyl 3-chlorobenzoate 81. The reaction of 3-pyridinepropanol 71 (21 mg, $0.150 \mathrm{mmol})$ according to the general procedure afforded $8 \mathrm{mg}(18 \%)$ of product 81 , isolated as a colorless oil; IR (neat) $\mathrm{cm}^{-1}$ : 3031, 2958, 2909, 2818, 1721, 1575, 1424, 1288, 1257, 1129, 1074, and 749; ${ }^{1} \mathrm{H}$ NMR $\left(400 \mathrm{MHz}, \mathrm{CDCl}_{3}\right): \delta 8.50(\mathrm{~s}, 1 \mathrm{H}), 8.47(\mathrm{~d}, J=4.8 \mathrm{~Hz}, 1 \mathrm{H}), 8.00-7.95$ $(\mathrm{m}, 1 \mathrm{H}), 7.93-7.87(\mathrm{~m}, 1 \mathrm{H}), 7.54(\mathrm{~d}, J=7.6 \mathrm{~Hz}, 2 \mathrm{H}), 7.40(\mathrm{t}, J=7.6 \mathrm{~Hz}, 1 \mathrm{H}), 7.26-7.20(\mathrm{~m}$, $1 \mathrm{H}), 4.37(\mathrm{t}, J=6.4 \mathrm{~Hz}, 2 \mathrm{H}), 2.85-2.75(\mathrm{~m}, 2 \mathrm{H})$, and $2.18-2.07(\mathrm{~m}, 2 \mathrm{H}) ;{ }^{13} \mathrm{C} \mathrm{NMR}(100 \mathrm{MHz}$, $\left.\mathrm{CDCl}_{3}\right): \delta 165.3,149.9,147.8,136.3,135.8,134.6,133.1,131.9,129.7,129.6,127.7,123.4,64.4$, 29.9, and 29.6; HRMS (ESI-positive): calcd for $\mathrm{C}_{15} \mathrm{H}_{14}{ }^{35} \mathrm{ClNO}_{2}([\mathrm{M}+\mathrm{H}])^{+}:$276.0786, found: 276.0786.

$N$-Benzyl 3-chlorobenzamide 10a [43]. The reaction of benzylamine 9a (16 $\mathrm{mg}, 0.150 \mathrm{mmol})$ according to the general procedure afforded $32 \mathrm{mg}(86 \%)$ of product $10 \mathrm{a}$, isolated as a white solid; ${ }^{1} \mathrm{H}$ NMR (500 MHz, $\left.\mathrm{CDCl}_{3}\right): \delta 7.77(\mathrm{~s}, 1 \mathrm{H}), 7.65(\mathrm{~d}, J=7.5 \mathrm{~Hz}, 1 \mathrm{H}), 7.51-7.43(\mathrm{~m}, 1 \mathrm{H})$, 7.41-7.28 (m, 6H), 6.44 (brs, $1 \mathrm{H})$, and $4.63(\mathrm{~d}, J=5.5 \mathrm{~Hz}, 2 \mathrm{H}) ;{ }^{13} \mathrm{C} \mathrm{NMR}\left(125 \mathrm{MHz}, \mathrm{CDCl}_{3}\right)$ : $\delta$ 166.0, 137.8, 136.2, 134.8, 131.6, 129.9, 128.8, 128.0, 127.8, 127.3, 125.0, and 44.3; HRMS (APCI-positive): calcd for $\mathrm{C}_{14} \mathrm{H}_{13} \mathrm{ClNO}([\mathrm{M}+\mathrm{H}])^{+}: 246.0686$, found: 246.0706 .

3-Chloro- $N$-isopropylbenzamide $\mathbf{1 0 b}$ [44]. The reaction of isopropylamine $\mathbf{9 b}$ (9 $\mathrm{mg}$, $0.150 \mathrm{mmol}$ ) according to the general procedure afforded $26 \mathrm{mg}(87 \%)$ of product $10 \mathrm{~b}$, 
isolated as a white solid; ${ }^{1} \mathrm{H}$ NMR $\left(400 \mathrm{MHz}, \mathrm{CDCl}_{3}\right): \delta 7.73(\mathrm{t}, J=1.8 \mathrm{~Hz}, 1 \mathrm{H}), 7.69-7.59(\mathrm{~m}$, $1 \mathrm{H}), 7.49-7.41(\mathrm{~m}, 1 \mathrm{H}), 7.35(\mathrm{t}, J=7.8 \mathrm{~Hz}, 1 \mathrm{H}), 6.03(\mathrm{br} . \mathrm{s}, 1 \mathrm{H}), 4.44-4.13(\mathrm{~m}, 1 \mathrm{H})$, and 1.26 $(\mathrm{d}, \mathrm{J}=6.8 \mathrm{~Hz}, 6 \mathrm{H}) ;{ }^{13} \mathrm{C}$ NMR $\left(100 \mathrm{MHz}, \mathrm{CDCl}_{3}\right): \delta 165.4,136.8,134.7,131.3,129.8,127.2$, 125.0, 42.1, and 22.8; HRMS (ESI-positive): calcd for $\mathrm{C}_{12} \mathrm{H}_{12}{ }^{35} \mathrm{ClNO}([\mathrm{M}+\mathrm{H}])^{+}:$198.0680, found: 198.0704 .

3-Chloro- $N$-cyclohexylbenzamide 10c [45]. The reaction of cyclohexylamine 9c (15 mg, $0.150 \mathrm{mmol}$ ) according to the general procedure afforded $22 \mathrm{mg}(65 \%)$ of product 10c, isolated as a white solid; IR (neat) $\mathrm{cm}^{-1}$ : 3322, 3070, 2929, 2853, 1633, 1539, 1327, 1081, 753; ${ }^{1} \mathrm{H} \mathrm{NMR}\left(400 \mathrm{MHz} \mathrm{CDCl}_{3}\right): \delta 7.75-7.71(\mathrm{~m}, 1 \mathrm{H}), 7.64-7.60(\mathrm{~m}, 1 \mathrm{H}), 7.47-7.42(\mathrm{~m}, 1 \mathrm{H}), 7.35$ $(\mathrm{t}, J=7.8 \mathrm{~Hz}, 1 \mathrm{H}), 6.06$ (brs, 1H), 4.03-3.89 (m, 1H), 2.07-1.98 (m, 2H), 1.81-1.71 (m, 2H), 1.70-1.62 (m, 1H), 1.49-1.35 (m, 2H), and 1.30-1.17 (m, 3H); ${ }^{3} \mathrm{C} \mathrm{NMR}\left(100 \mathrm{MHz}, \mathrm{CDCl}_{3}\right): \delta$ 165.3, 136.9, 134.7, 131.3, 129.8, 127.2, 125.0, 48.9, 33.2, 25.5, and 24.9; HRMS (ESI-positive): calcd for $\mathrm{C}_{13} \mathrm{H}_{16} \mathrm{ClNO}([\mathrm{M}+\mathrm{H}])^{+}$: 238.0993, found: 238.1009 .

$N$-(tert-butyl)-3-chlorobenzamide 10d [46]. The reaction of tert-butyl amine 9d (11 mg, $0.150 \mathrm{mmol})$ according to the general procedure afforded $14 \mathrm{mg}(43 \%)$ of product $\mathbf{1 0 d}$, isolated a white solid; ${ }^{1} \mathrm{H}$ NMR $\left(400 \mathrm{MHz}, \mathrm{CDCl}_{3}\right): \delta 7.69(\mathrm{t}, J=2.0 \mathrm{~Hz}, 1 \mathrm{H}), 7.61-7.56(\mathrm{~m}$, 1H), 7.46-7.42 (m, 1H), $7.35(\mathrm{t}, J=7.8 \mathrm{~Hz}, 1 \mathrm{H}), 5.92(\mathrm{brs}, 1 \mathrm{H})$, and $1.47(\mathrm{~s}, 9 \mathrm{H}) ;{ }^{13} \mathrm{C} \mathrm{NMR}$ $\left(100 \mathrm{MHz} \mathrm{CDCl}_{3}\right): \delta 165.5,137.8,134.6,131.1,129.8,127.1,124.9,51.9$, and 28.8; HRMS (ESI-positive): calcd for $\mathrm{C}_{11} \mathrm{H}_{14} \mathrm{ClNO}([\mathrm{M}+\mathrm{H}])^{+}: 212.0837$, found: 212.0853 .

\section{Conclusions}

In conclusion, we have developed novel benziodazolone compounds readily prepared from iodobenzamides using $m \mathrm{CPBA}$, and the solid structure was confirmed by X-ray crystallography. These new benziodazolones can act as coupling assistant reagents to alcohols and amines, and their ligands can act as a coupling partner to give the corresponding ester and amides in moderate to good yields. In addition, it was found that the newly synthesized benzoiodazolone demonstrated better efficiency than the corresponding benziodoxolone in the esterification reaction.

Supplementary Materials: The following are available online, the ${ }^{1} \mathrm{H}$ and ${ }^{13} \mathrm{C}$ NMR spectra of $\mathbf{5 a}-\mathbf{j}$, 6a-j, 8a-1, 10a-d, 11, Figure S1: X-ray crystallography data of 5a, Figure S2: Mass study.

Author Contributions: Conceptualization, A.Y.; methodology, A.Y. and M.T.S.; investigation, M.T.S.; resources, M.T.S., Y.A.V., P.S.P. and M.S.Y.; data analysis, A.Y., M.T.S. and A.S.; Data Discussion, A.Y., M.T.S., Y.A.V., P.S.P. and A.S., X-ray analysis, G.T.R.; writing-original draft preparation, A.Y., V.V.Z. and A.S.; writing-review and editing, A.Y., M.T.S., A.S. and G.T.R.; supervision, A.Y.; project administration, A.Y. and A.S.; funding acquisition, M.S.Y., V.V.Z. and A.S. All authors have read and agreed to the published version of the manuscript.

Funding: This research was funded by the National Science Foundation, grant number CHE-1759798, JSPS Fund for the Promotion of Joint International Research (Grant No 16KK0199), JST CREST (No. JRMJCR19R2), and a research grant from the Russian Science Foundation (RSF-21-73-20031).

Institutional Review Board Statement: Not applicable.

Informed Consent Statement: Not applicable.

Data Availability Statement: Data for the compounds are included in the article and the Supplementary Materials.

Conflicts of Interest: The authors declare no conflict of interest.

\section{References}

1. Olofsson, B.; Ilan, M.; Rappoport, Z. Patai's The Chemistry of Hypervalent Halogen Compounds; John Wiley \& Sons: Chichester, UK, 2019.

2. Wirth, T. (Ed.) Hypervalent Iodine Chemistry; Springer International Publishing: Cham, Switzerland, 2016.

3. Zhdankin, V.V. Hypervalent Iodine Chemistry: Preparation, Structure, and Synthetic Applications of Polyvalent Iodine Compounds; Wiley: Chichester, UK, 2013. 
4. Hari, D.P.; Caramenti, P.; Waser, J. Cyclic Hypervalent Iodine Reagents: Enabling Tools for Bond Disconnection via Reactivity Umpolung. Acc. Chem. Res. 2018, 51, 3212-3225. [CrossRef] [PubMed]

5. Li, Y.; Hari, D.P.; Vita, M.V.; Waser, J. Cyclic Hypervalent Iodine Reagents for Atom-Transfer Reactions: Beyond Trifluoromethylation. Angew. Chem. Int. Ed. 2016, 55, 4436-4454. [CrossRef]

6. Robidas, R.; Legault, C.Y. Cyclic Haloiodanes: Syntheses, Applications and Fundamental Studies. Helv. Chim. Acta 2021, 104, e2100111. [CrossRef]

7. Vlasenko, Y.; Yusubov, M.S.; Shafir, A.; Postnikov, P.S. Hypervalent iodine in the structure of N-heterocycles: Synthesis, structure, and application in organic synthesis. Chem. Heterocycl. Compd. 2020, 56, 854-866. [CrossRef]

8. Yoshimura, A.; Zhdankin, V.V. Advances in Synthetic Applications of Hypervalent Iodine Compounds. Chem. Rev. 2016, 116, 3328-3435. [CrossRef] [PubMed]

9. Zhang, C.; Yang, X.-G.; Hu, Z.-N.; Jia, M.-C.; Du, F.-H. Recent Advances and the Prospect of Hypervalent Iodine Chemistry. Synlett 2021, 32, 1289-1296. [CrossRef]

10. Declas, N.; Pisella, G.; Waser, J. Vinylbenziodoxol(on)es: Synthetic Methods and Applications. Helv. Chim. Acta 2020, 103, e2000191. [CrossRef]

11. Waser, J. Benziodoxol(on)e Reagents as Tools in Organic Synthesis: The Background behind the Discovery at the Laboratory of Catalysis and Organic Synthesis. Synlett 2016, 27, 2761-2773. [CrossRef]

12. Charpentier, J.; Früh, N.; Togni, A. Electrophilic Trifluoromethylation by Use of Hypervalent Iodine Reagents. Chem. Rev. 2014, 115, 650-682. [CrossRef] [PubMed]

13. Le Vaillant, F.; Waser, J. Alkynylation of radicals: Spotlight on the "Third Way" to transfer triple bonds. Chem. Sci. 2019, 10, 8909-8923. [CrossRef]

14. Zhdankin, V.V. Benziodoxole-Based Hypervalent Iodine Reagents in Organic Synthesis. Curr. Org. Synth. 2005, 2, 121-145. [CrossRef]

15. Tian, J.; Gao, W.-C.; Zhou, D.-M.; Zhang, C. Recyclable Hypervalent Iodine(III) Reagent Iodosodilactone as an Efficient Coupling Reagent for Direct Esterification, Amidation, and Peptide Coupling. Org. Lett. 2012, 14, 3020-3023. [CrossRef] [PubMed]

16. Gao, W.-C.; Zhang, C. Synthesis, characterization, and initial reaction study of two new bicyclic hypervalent iodine(III) reagents. Tetrahedron Lett. 2014, 55, 2687-2690. [CrossRef]

17. Zhang, C.; Liu, S.-S.; Sun, B.; Tian, J. Practical Peptide Synthesis Mediated by a Recyclable Hypervalent Iodine Reagent and Tris(4-methoxyphenyl)phosphine. Org. Lett. 2015, 17, 4106-4109. [CrossRef]

18. Liu, D.; Guo, Y.-L.; Qu, J.; Zhang, C. Recyclable hypervalent-iodine-mediated solid-phase peptide synthesis and cyclic peptide synthesis. Beilstein J. Org. Chem. 2018, 14, 1112-1119. [CrossRef] [PubMed]

19. Qiu, L.-J.; Liu, D.; Zheng, K.; Zhang, M.; Zhang, C. A Benziodoxole-Based Hypervalent Iodine(III) Compound Functioning as a Peptide Coupling Reagent. Front. Chem. 2020, 8, 183. [CrossRef]

20. Zhdankin, V.V.; Arbit, R.M.; McSherry, M.; Mismash, B.; Young, V.G. Structure and chemistry of acetoxybenziodazole. Ac-idcatalyzed rearrangement of benziodazoles to 3-iminobenziodoxoles. J. Am. Chem. Soc. 1997, 119, 7408-7409. [CrossRef]

21. Zhdankin, V.V.; Koposov, A.E.; Smart, J.T.; Tykwinski, R.R.; McDonald, R.; Morales-Izquierdo, A. Secondary Bonding-Directed Self-Assembly of Amino Acid Derived Benziodazoles: Synthesis and Structure of Novel Hypervalent Iodine Macrocycles. J. Am. Chem. Soc. 2001, 123, 4095-4096. [CrossRef]

22. Zhdankin, V.V.; Arbit, R.M.; Lynch, B.; Kiprof, P.; Young, J.V.G. Structure and Chemistry of Hypervalent Iodine Heterocycles: Acid-Catalyzed Rearrangement of Benziodazol-3-ones to 3-Iminiumbenziodoxoles. J. Org. Chem. 1998, 63, 6590-6596. [CrossRef]

23. Zhdankin, V.V.; Koposov, A.Y.; Su, L.S.; Boyarskikh, V.V.; Netzel, B.C.; Young, V.G. Synthesis and structure of amino ac-id-derived benziodazoles: New hypervalent iodine heterocycles. Org. Lett. 2003, 5, 1583-1586. [CrossRef]

24. Hari, D.P.; Schouwey, L.; Barber, V.; Scopelliti, R.; Fadaei-Tirani, F.; Waser, J. Ethynylbenziodazolones (EBZ) as Electrophilic Al-kynylation Reagents for the Highly Enantioselective Copper-Catalyzed Oxyalkynylation of Diazo Compounds. Chem. Eur. J. 2019, 25, 9522-9528. [CrossRef] [PubMed]

25. Yang, X.-G.; Zheng, K.; Zhang, C. Electrophilic Hypervalent Trifluoromethylthio-Iodine(III) Reagent. Org. Lett. 2020, 22, 2026-2031. [CrossRef]

26. Ren, J.; Du, F.; Jia, M.; Hu, Z.; Chen, Z.; Zhang, C. Ring Expansion Fluorination of Unactivated Cyclopropanes Mediated by a New Monofluoroiodane(III) Reagent. Angew. Chem. Int. Ed. 2021, 60, 24171-24178. [CrossRef] [PubMed]

27. Gu, H.; Wang, C. Rhenium-catalyzed dehydrogenative olefination of C(sp3)-H bonds with hypervalent iodine(iii) reagents. Org. Biomol. Chem. 2015, 13, 5880-5884. [CrossRef]

28. Yoshimura, A.; Shea, M.T.; Makitalo, C.L.; Jarvi, M.E.; Rohde, G.T.; Saito, A.; Yusubov, M.S.; Zhdankin, V.V. Preparation, structure, and reactivity of bicyclic benziodazole: A new hypervalent iodine heterocycle. Beilstein J. Org. Chem. 2018, 14, 1016-1020. [CrossRef]

29. Brotzel, F.; Kempf, B.; Singer, T.; Zipse, H.; Mayr, H. Nucleophilicities and Carbon Basicities of Pyridines. Chem. Eur. J. 2006, 13, 336-345. [CrossRef]

30. Ochiai, M.; Sueda, T.; Miyamoto, K.; Kiprof, P.; Zhdankin, V.V. trans influence on hypervalent bonding of aryl $\lambda 3$-iodanes: Their stabilities and isodesmic reactions of benziodoxolones and benziodazolones. Angew. Chem. Int. Ed. 2006, 45, 8203-8206. [CrossRef] 
31. Qiu, F.-C.; Yang, W.-C.; Chang, Y.-Z.; Guan, B. Palladium-Catalyzed ortho -Halogenation of Tertiary Benzamides. Asian J. Org. Chem. 2017, 6, 1361-1364. [CrossRef]

32. Sperger, C.A.; Fiksdahl, A. Gold-Catalyzed Tandem Cyclizations of 1,6-Diynes Triggered by Internal N- and O-Nucleophiles. J. Org. Chem. 2010, 75, 4542-4553. [CrossRef] [PubMed]

33. Pacula, A.J.; Scianowski, J.; Aleksandrzak, K.B. Highly efficient synthesis and antioxidant capacity of N-substituted benzisoselenazol-3(2H)-ones. RSC Adv. 2014, 4, 48959-48962. [CrossRef]

34. Yakura, T.; Fujiwara, T.; Yamada, A.; Nambu, H. 2-Iodo-N-isopropyl-5-methoxybenzamide as a highly reactive and environmentally benign catalyst for alcohol oxidation. Beilstein J. Org. Chem. 2018, 14, 971-978. [CrossRef]

35. Evans, M.B.; Haken, J.K. Dispersion and selectivity indexes in gas chromatography. III. Alkyl, $\omega$-chloroethyl and alkenyl benzoate and chlorobenzoate esters. J. Chromatogr. 1989, 462, 31-37. [CrossRef]

36. Mukhopadhyay, S.; Batra, S. Direct Transformation of Arylamines to Aryl Halides via Sodium Nitrite and N -Halosuccinimide. Chem. Eur. J. 2018, 24, 14622-14626. [CrossRef]

37. Molander, G.A.; Cavalcanti, L.N. Metal-Free Chlorodeboronation of Organotrifluoroborates. J. Org. Chem. 2011, 76, 7195-7203. [CrossRef] [PubMed]

38. De Sarkar, S.; Grimme, S.; Studer, A. NHC Catalyzed Oxidations of Aldehydes to Esters: Chemoselective Acylation of Alcohols in Presence of Amines. J. Am. Chem. Soc. 2010, 132, 1190-1191. [CrossRef]

39. Wybon, C.C.D.; Mensch, C.; Hollanders, C.; Gadais, C.; Herrebout, W.A.; Ballet, S.; Maes, B.U.W. Zn-Catalyzed tert-Butyl Nic-otinate-Directed Amide Cleavage as a Biomimic of Metallo-Exopeptidase Activity. ACS Catal. 2018, 8, 203-218. [CrossRef]

40. Simeonov, S.; Simeonov, A.P.; Todorov, A.R.; Kurteva, V.B. Enantioresolution of a Series of Chiral Benzyl Alcohols by HPLC on a Dinitrobenzoylphenylglycine Stationary Phase after Achiral Pre-Column Derivatization. Am. J. Anal. Chem. 2010, 1, 1-13. [CrossRef]

41. Huston, R.C.; Robinson, K.R. Chloro-substituted diphenylmethanes, phenyl benzyl ethers, and benzophenones prepared from oor p-cresol. J. Am. Chem. Soc. 1951, 73, 2483-2486. [CrossRef]

42. Muñiz, K.; García, B.; Martínez, C.; Piccinelli, A. Dioxoiodane Compounds as Versatile Sources for Iodine(I) Chemistry. Chem. Eur. J. 2016, 23, 1539-1545. [CrossRef] [PubMed]

43. Vanjari, R.; Guntreddi, T.; Singh, K.N. MnO2 Promoted Sequential C-O and C-N Bond Formation via C-H Activation of Methylarenes: A New Approach to Amides. Org. Lett. 2013, 15, 4908-4911. [CrossRef] [PubMed]

44. Sharma, S.; Park, E.; Park, J.; Kim, I.S. Tandem Rh(III)-Catalyzed Oxidative Acylation of Secondary Benzamides with Aldehydes and Intramolecular Cyclization: The Direct Synthesis of 3-Hydroxyisoindolin-1-ones. Org. Lett. 2012, 14, 906-909. [CrossRef] [PubMed]

45. Nandi, J.; Vaughan, M.Z.; Sandoval, A.L.; Paolillo, J.M.; Leadbeater, N.E. Oxidative Amidation of Amines in Tandem with Transamidation: A Route to Amides Using Visible-Light Energy. J. Org. Chem. 2020, 85, 9219-9229. [CrossRef] [PubMed]

46. Park, J.; Park, S.; Jang, G.S.; Kim, R.H.; Jung, J.; Woo, S.K. Weak base-promoted selective rearrangement of oxaziridines to amides via visible-light photoredox catalysis. Chem. Commun. 2021, 57, 9995-9998. [CrossRef] [PubMed] 